“C 2017 IEEE. Personal use of this material is permitted. Permission from IEEE must be obtained for all other uses, in any current or future media, including reprinting/republishing this material for advertising or promotional purposes, creating new collective works, for resale or redistribution to servers or lists, or reuse of any copyrighted component of this work in other works." 


\title{
Deep Additive Least Squares Support Vector Machines for Classification with Model Transfer
}

\author{
Guanjin Wang ${ }^{1,2}$, Guangquan Zhang ${ }^{1}$, Kup-Sze Choi ${ }^{2}$, and Jie Lu ${ }^{1}$ \\ ${ }^{1}$ Centre for Artificial Intelligence, School of Software, Faculty of Engineering and Information Technology, \\ University of Technology Sydney, Broadway, NSW, 2007, Australia \\ Email: Guanjin.Wang@student.uts.edu.au, Jie.Lu, Guangquan.Zhang@uts.edu.au \\ ${ }^{2}$ Centre for Smart Health, School of Nursing, \\ The Hong Kong Polytechnic University, Hong Kong, China \\ Email: thomasks.choi@polyu.edu.hk
}

\begin{abstract}
The additive kernel least squares support vector machine (AK-LS-SVM) has been well used in classification tasks due to its inherent advantages. For example, additive kernels work extremely well for some specific tasks, such as computer vision classification, medical research, and some specialized scenarios. Moreover, the analytical solution using AK-LS-SVM can formulate leave-one-out cross-validation error estimates in a closed form for parameter tuning, which drastically reduces the computational cost and guarantee the generalization performance especially on small and medium datasets. However, AK-LS-SVM still faces two main challenges: (1) improving the classification performance of AK-LS-SVM, and (2) saving time when performing a grid search for model selection. Inspired by the stacked generalization principle and the transfer learning mechanism, a layer-by-layer combination of AK-LS-SVM classifiers embedded with transfer learning is proposed in this study. This new classifier is called deep transfer additive kernel least square support vector machine (DTA-LS-SVM) which overcomes these two challenges. Also, considering that imbalanced datasets are involved in many real-world scenarios, especially for medical data analysis, the deep-transfer element is extended to compensate for this imbalance, thus leading to the development of another new classifier iDTA-LS-SVM. In the hierarchical structure of both DTA-LS-SVM and iDTA-LS-SVM, each layer has an AKLS-SVM and the predictions from the previous layer act as an additional input feature for the current layer. Importantly, transfer learning is also embedded to guarantee generalization consistency between the adjacent layers. Moreover, both iDTALS-SVM and DTA-LS-SVM can ensure the minimal leave-oneout error by using the proposed fast leave-one-out cross validation strategy on the training set in each layer. We compared the proposed classifiers DTA-LS-SVM and IDTA-LS-SVM with the traditional LS-SVM and SVM using additive kernels on seven public UCI datasets and one real world dataset. The experimental results show that both DTA-LS-SVM and iDTA-LS-SVM exhibit better generalization performance and faster learning speed.
\end{abstract}

Index Terms-Classification, deep architectures, support vector machine, transfer learning

\section{INTRODUCTION}

The additive kernel least squares support vector machine (AK-LS-SVM) has been well used in many classification tasks due to its distinct advantages. For example, the generalization performances of kernel-based learning methods depend highly on the parameter selection, such as the model selection of good values for regularization and kernel parameters. To make an unbiased selection, parameter selection strategies based on the minimisation of the leave-one-out cross validation estimate is usually preferred but with unavoidably expensive computation. However, since the analytical solution of AK-LSSVM can formulate a simple yet effective leave-one-out cross validation estimate in a closed form, it only has a negligible additional computational cost [1]. This particularly benefits real world applications with small or medium size datasets. For example, many medical applications involve small or medium datasets due to the complex and/or expensive data collection processes and the concerns of the patients' privacy. Therefore, it becomes necessary for us to minimize the leave-one-out error on these datasets, which is an almost unbiased estimator of the generalization error [1]. In these scenarios LS-SVM can improve the generalization performances by using the fast leave-one-out cross validation estimate. Also, in terms of additive kernels in AK-LS-SVM, a kernel is additive if it can be broken down into a sum of kernel functions of each dimension [2], i.e.,

$$
k(\boldsymbol{x}, \boldsymbol{y})=\sum_{i=1}^{d} k\left(x_{i}, y_{i}\right)
$$

Additive kernels are widely used in different classification and regression tasks [3]-[5], such as the histogram intersection kernel in Eq. (2), the $\chi^{2}$ kernel in Eq. (3) and the additive Gaussian kernel used in this work (see Eq. (6)).

$$
\begin{gathered}
k_{h i s t}(\boldsymbol{x}, \boldsymbol{y})=\sum_{i=1}^{d} \min \left(x_{i}, y_{i}\right) \\
k_{\chi^{2}}(\boldsymbol{x}, \boldsymbol{y})=\frac{2 x_{i} y_{i}}{x_{i}+y_{i}}
\end{gathered}
$$

In addition, additive kernels exhibit their advantages when tackling special scenarios. For example, they have been used in handling missing data in community health studies [6], where only the kernel functions of each dimension without missing values are summed together. In [7], additive kernels are expected to well represent the distance/similarity measures for medical data with mixed types of features, such as features that change over time or features that do not change. 
Based on the above summary, we found that the application of AK-LS-SVM has huge potential in the real world. However, it still faces two main challenges: (1) The classification performance of AK-LS-SVM is comparatively low, and (2) the grid search for good values for model selection is still time consuming. The aim of this current work is to develop a stacked hierarchical AK-LS-SVM classifier, i.e., deep transfer additive least square support vector machine (DTA-LS-SVM), by integrating the stacked generalization principle and the transfer learning mechanism, to overcome these two challenges. Furthermore, many real-world scenarios involve imbalanced datasets. For example, in medicine, there may be only a few examples of patients diagnosed as having contracted diseases compared to those who are healthy. We consider these scenarios and extend DTA-LS-SVM to iDTA-LS-SVM for handling imbalanced datasets. In the proposed hierarchical structure, each layer has an AK-LS-SVM. Following the stacked generalization principle, the original data inputs and the predictions from the previous layer become the new data input of the next layer to enhance the performance of the whole stacked architecture. Moreover, transfer learning is used to guarantee a consistency between the adjacent layers such that the previously learned model knowledge from the previous layer can be leveraged for model construction in the adjacent higher layer to further improve the generalization capability. The contributions of this work can be summarized below:

1) The novel classifiers DTA-LS-SVM and iDTA-LS-SVM proposed can significantly enhance the generalization performance of AK-LS-SVM respectively on balanced and imbalanced datasets. Following the stacked generalization principle, the proposed classifiers organize multiple modules in a layerby-layer structure in which every module contains an AK-LSSVM. From the second layer, the data input space for each layer comprises the original data features and the predictions from the previous layer as an appended feature. Through this deep stacking architecture, the appended feature space helps open the manifold structure in the original data space in a stacked way so as to achieve the classification performance improvement.

2) Transfer learning is embedded from the second layer in the proposed classifiers DTA-LS-SVM and iDTA-LS-SVM to ensure that the consistency across adjacent layers is guaranteed and the classification capability of the higher layer can be further enhanced. Moreover, the kernel and regularization parameters are randomly selected in DTA-LS-SVM and iDTALS-SVM, and AK-LS-SVM with transfer learning in each layer to ensure the minimal leave-one-out error on the training set of each layer is achieved by using the proposed fast leaveone-out cross validation strategy.

3) The experimental results show that both DTA-LS-SVM and iDTA-LS-SVM give improved generalization performance and fast learning speed on seven balanced and imbalanced UCI datasets and one real community healthcare dataset, compared with the traditional least square support vector machine (LSSVM) and support vector machine (SVM) using the same additive kernels.

The remainder of this paper is organized as follows. The related work is introduced in Section II. In Section III the proposed classifier DTA-LS-SVM is presented. In particular, a fast leave-one-out cross validation strategy for parameter tuning is introduced. In Section IV, the experimental results are provided on the seven UCI datasets and one real community healthcare dataset. Finally, the conclusions and future work are given in Section $\mathrm{V}$.

\section{RELATED WORK}

In this section some background knowledge of LS-SVM and AK-LS-SVM is described because our work involves both proposed classifiers integrated with transfer learning in a deep structure.

\section{A. Shallow and deep architecture}

Classifiers in shallow architecture traditionally consist of an input layer, a single processing layer and an output layer. For example, LS-SVM and AK-LS-SVM used in this work have a layer of kernel function which is applied to the input, followed by a linear combination of the kernel outputs. Such shallow machines have been widely used in classification and regression with good performances [8] [9] [10]. However, shallow architectures have problems in providing an efficient representation for certain types of functions. Deep structures are known to comprise several layers of non-linear operations, such as in the neural network with many hidden layers, which aim to learn hierarchical representations. Benefiting from the multiple levels of representation and abstraction, deep architectures learn the complicated functions mapping the input to the output of the data.

Using shallow classifiers like SVM in combination with deep architectures has been proposed in the literature. For example, Abdullah et al. [11] presented a deep support vector machine (D-SVM), which used the non-linear combinations of kernel activations of support vectors as inputs to train a SVM on the upper layer. Moreover, the deep SVMs were combined with the product rule ensemble for combining multiple image descriptors. Tang [12] used linear SVMs rather than a softmax activation function in convolutional neural networks (CNNs) to learn to minimize a margin-based loss instead of the crossentropy loss. In this work, we focus on the least squares version of SVM in deep architecture.

\section{B. Deep Stacked architecture}

There are different types of deep architectures, such as CNNs [13], deep belief networks (DBNs) [14], deep Boltzmann machines (DBM) [15] and deep auto encoders [16] etc. In this work, the proposed classifier DTA-LS-SVM is built in a deep stacked architecture [17], which is trained in a supervised and module-wise fashion. Unlike other deep architectures such as DBNs, it does not employ back propagation over all modules and does not aim to find the transformed feature representation. To learn complex functions effectively, it stacks multiple modules in a chain, and the outputs from the previous layer are fed into the inputs of the module of the next layer. Due to this nature of building a hierarchy of simplified modules trained by itself, deep stacked architecture is relatively 


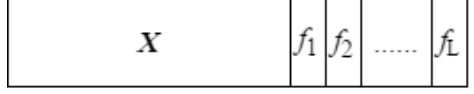

(a)

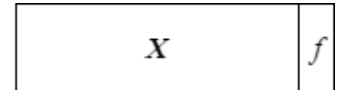

(b)
Fig. 1: Two ways to expand the feature space in deep stacked architecture. (a) $f_{l}$ is the prediction from the previous module $l$. (b) $f$ changes with the current module layer. For example, in module $2, f=f_{1}$, in module $3, f=f_{2}, \cdots$, in module $L$, $f=f_{L-1}$.

simple and easy to implement. Through the deep stacked architecture, the appended feature space opens the original data manifolds. This architecture follows the philosophy of "stacked generalization [18]" which indeed enhances classification performances and improves generalization in learning complex functions. In order to learn complex functions or classifiers effectively, it stacks multiple modules in a chain, and the outputs from the module(s) from the previous layer are fed into the inputs of the module of the next layer. Deep stacked architecture was first introduced in 2011 [17]. After that, a kernel version DSNs (K-DSNs) was proposed by Deng et al. [19], which integrates deep learning and kernel learning. By using the kernel trick, the hidden neurons in each DSN layer becomes infinity. Another novel DSNs, which is called tensor-DSNs (T-DSNs) was presented by Hutchinson et al. [20]. In this method, each module has a bilinear mapping from two hidden layers to the output layer by incorporating higher order statistics of the hidden binary features through a weight tensor. Vinyals et al. [21] proposed a recursive perceptual representation using layers of linear SVMs and incorporating with random projections of weak predictions from each layer.

Through the deep stacked architecture, the appended feature space using the predictions from the module(s) of the previous layers open the original data manifolds such that the generalization performances may be improved. There are two ways to append the feature space with the increase of the depth in deep stacking architecture. As depicted in Fig. 1(a), the new feature space for the module in the higher layer comes from the concatenation of the predicted outputs from all the modules of the previous layers and the original input features. The mentioned work above belongs to this category. Fig 1(b) illustrates a different type to append feature space on which our work concentrates. In our work, the new feature space for the module of the higher layer comes from the concatenation of the predicted outputs, that is, from the module of the original input features and the predicted outputs only for the module of the previous layer.

\section{Transfer learning}

Transfer learning has been another important research topic in machine learning. Given a source domain $D_{S}$ and its learning task $T_{S}$, a target domain $D_{T}$ and its learning task $T_{T}$, The goal of transfer learning is to help improve the learning process in $D_{T}$ by leveraging the knowledge in $D_{S}$, when $D_{S} \neq D_{T}$, or $T_{S} \neq T_{T}$. In our scenario, from the bottom to top in the deep stacked architecture, the module of the previous layer is used as the source domain, and the adjacent module of next layer is used as the target domain.

In the proposed deep stacking architecture, the adjacent models always have the same original feature space, and an additional feature is taken as the appended feature for the predictions of the adjacent layers to reflect the discriminative information about the classes in the original feature space. Therefore, we can postulate that there is a certain consistency between $D_{S}$ and $D_{T}$ such that transfer learning between the adjacent layers can be introduced. In other words, it is worthwhile embedding transfer learning into the deep stacking architecture, which uses previously learned knowledge from $D_{S}$ to help the learning process in $D_{T}$.

To deploy transfer learning, the main question is "what to transfer". It asks which part of knowledge is used to transfer across domains or tasks. From the literature, the leveraged knowledge can be categorized into instances-, feature-, or model/parameters-based transfer learning [22]. The instance-transfer approach re-weights certain data points in the source domain for use in the target domain [23], [24]. The feature-representation-transfer approach encodes the transferred knowledge across domains into a shared representative feature structure, and the target model is guided in the new feature space [25], [26]. The model/parametertransfer approach assumes that the source and target domains share parameters to some extent or prior distributions of the classifier [27], [28]. For readers who are interested, the following articles are suggested for further investigation: [22], [29], [30]. In this work, the transfer learning used belongs to the model/parameters-based transfer category.

\section{THE PROPOSED DEEP TRANSFER LEAST SQUARES SUPPORT VECTOR MACHINE}

We use $\boldsymbol{S}=\left\{\left(\boldsymbol{x}_{1}, y_{1}\right), \cdots,\left(\boldsymbol{x}_{N}, y_{N}\right)\right\}$ to represent the dataset adopted in this work. The input set is denoted as $\boldsymbol{X}$ and the corresponding output set is denoted as $\boldsymbol{Y} . \boldsymbol{x}_{i}=$ $\left(x_{1}^{i}, x_{2}^{i}, \cdots, x_{d}^{i}\right) \in \boldsymbol{X} \subset \boldsymbol{R}^{d}, y_{i} \in\{+1,-1\}$, and each sample $\boldsymbol{x}_{i}(i=1,2, \cdots, N)$ contains $d$ features. The general framework of DTA-LS-SVM is illustrated in Fig. 2. It can be seen that the proposed approach is built with multiple layers of AK-LS-SVM based modules in a stacked structure. The original data are given as data inputs to layer 1. For each module from layer 2, a transfer based AK-LS-SVM is trained using the transformed data with the original features and the appended feature where values are the previous layers' predictions. Moreover, model transfer is embedded to leverage relevant model knowledge from the adjacent module of the previous layer.

In the whole framework, the stacked generalization principle and the unfolding of manifold structure existing in the original data input space by concatenating the predictions from the adjacent module of the previous layer guarantee the improvement on the generalization capability. Moreover, transfer learning is used to maintain similarity across the adjacent modules by leveraging the previously learned knowledge from the previous module such that the generlization capability also can be improved as well. 


\section{A. AK-LS-SVM and its Adaptive Regularization}

AK-LS-SVM is based on statistical learning theory which is formulated on the structural risk minimization (SRM) principle. The learning process can be formalized as an optimization problem that minimizes the structural risk as follows:

$$
\Omega(f)+C \sum_{i=1}^{N} R_{e m p}\left(f\left(\boldsymbol{x}_{\boldsymbol{i}}\right), y_{i}\right)
$$

where $\Omega(f)$ is a regularization term that encodes some notion of smoothness for $f$ and prevent overfitting. $R_{e m p}$ is a chosen convex loss function that evaluates the quality of $f$ on the instance $\left\{\boldsymbol{x}_{i}, y_{i}\right\}$. $C$ gives a trade-off between the minimization of $R_{e m p}$ and the smoothness or simplicity enforced by a small $\Omega(f)$.

In the first processing layer $L_{1}$ in the framework of DTALS-SVM, a traditional AK-LS-SVM is used to find an optimal hyperplane $f(\boldsymbol{x})=\boldsymbol{w}^{T} \varphi(\boldsymbol{x})+b$. The regularization term is set to be $\Omega(f)=\frac{1}{2}\|\boldsymbol{w}\|^{2}$ and the loss function to be the weighted square loss $R_{e m p}\left(f(\boldsymbol{x})_{i}, y_{i}\right)=\left(f\left(\boldsymbol{x}_{i}\right)-y_{i}\right)^{2}$. Therefore, the learning problem in Eq. (4) becomes

$$
\begin{array}{ll}
\min _{\boldsymbol{w}, b} J(\boldsymbol{w}, b)= & \frac{1}{2}\left\|\boldsymbol{w}_{1}\right\|^{2}+\frac{C_{1}}{2} \sum_{i=1}^{N} \xi_{i 1}^{2} \\
\text { s.t. } & y_{i}=\boldsymbol{w}_{1}^{T} \varphi\left(\boldsymbol{x}_{i 1}\right)+b_{1}+\xi_{i 1} \\
& i=1,2, \ldots, n
\end{array}
$$

where $C$ is the regularization parameter and $\varphi\left(\boldsymbol{x}_{i 1}\right)=$ $\left(\tilde{\varphi}\left(x_{11}^{i}\right), \tilde{\varphi}\left(x_{21}^{i}\right), \cdots, \tilde{\varphi}\left(x_{k 1}^{i}\right), \cdots, \tilde{\varphi}\left(x_{d 1}^{i}\right)\right)$, and $\varphi\left(\boldsymbol{x}_{i 1}\right)$ is a feature mapping such that the additive kernel $\boldsymbol{K}$ below can be adopted in Eq. (5):

$$
\boldsymbol{K}\left(\boldsymbol{x}_{i 1}, \boldsymbol{x}_{j 1}\right)=\varphi\left(\boldsymbol{x}_{i 1}\right)^{T} \varphi\left(\boldsymbol{x}_{j 1}\right)=\sum_{k=1}^{d} k\left(x_{k}^{i 1}, x_{k}^{j 1}\right)
$$

In this work, an additive Gaussian kernel [5] is adopted, i.e., $k\left(x_{k}^{i 1}, x_{k}^{j 1}\right)=e^{\frac{-\left(x_{k}^{i 1}-x_{k}^{j 1}\right)}{\delta^{2}}}$, where $\delta$ is the kernel width.

From the next processing layer $\left.L_{l},(l=2,3, \cdots, L)\right)$, transfer learning is used to leverage model knowledge from $D_{S(l-1)}$ (source domain) to $D_{T l}$ (target domain), For example, first, the optimal $\boldsymbol{w}_{S(l-1)}$ is found by minimizing Eq. (5) in $D_{S(l-1)}$, then when $D_{T l}$ is encountered, a model is constructed in which $\boldsymbol{w}_{T l}$ gets as close as possible to the known $\boldsymbol{w}_{S(l-1)}$. Through editing the regularization term, the learning classification task from the second processing layer becomes the corresponding transfer based AK-LS-SVM, i.e.,

$$
\frac{1}{2}\left\|\boldsymbol{w}_{T l}-\boldsymbol{w}_{S(l-1)}\right\|^{2}+\frac{C_{l}}{2} \sum_{i=1}^{N} \xi_{i l}^{2}
$$

Moreover, the regularization term can be further revised to $\left\|\boldsymbol{w}_{T l}-\lambda_{l} \boldsymbol{w}_{S(l-1)}\right\|$ by adding the weighting factor $\lambda_{l}$ to control the degree to which the new model is close to the source model. This evaluates the similarity between $\boldsymbol{w}_{S(l-1)}$ and $\boldsymbol{w}_{T l}$ in the optimization problem above.
B. Transfer learning in DTA-LS-SVM and its fast leave-oneout cross validation

In this section, we describe in detail how to use transfer learning across modules in DTA-LS-SVM. As demonstrated in Fig 2, the proposed classifier is built by multiple layers of modules, which from the second module, each learns a transfer based AK-LS-SVM on the original data inputs with an additional feature whose values are the predictions from the module of the previous layer. The core motivation of DTA-LSSVM is that when the deeper structure is well trained, it tends to do a better job at disentangling the underlying factors of variation [31]. The recursive leverage of previous predictions helps to move apart the manifolds in the original data in a stacked way such that a better separability can be achieved [21].

As mentioned in III-A, in the first processing layer $L_{1}$, a traditional AK-LS-SVM model is constructed using the additive kernel defined in Eq. (5) giving the decision function $f_{1}\left(\boldsymbol{x}_{i}\right)=\boldsymbol{w}_{1}^{T} \varphi\left(\boldsymbol{x}_{i}\right)+b_{1}$. Also the predicted label vector $\boldsymbol{F}_{1}$ of $\boldsymbol{X}$ is obtained, where $\boldsymbol{F}_{1}=\left(f_{1}\left(\boldsymbol{x}_{1}\right), f_{1}\left(\boldsymbol{x}_{2}\right), \cdots, f_{1}\left(\boldsymbol{x}_{N}\right)\right)$.

From the processing layer $L_{l}(l=2,3, \ldots L)$, the new data input $\boldsymbol{X}_{l}^{\prime}$ is the augmentation of the original data input set $\boldsymbol{X}$ and the predicted label vector $\boldsymbol{F}_{l-1}$ from the previous layer, which can be denoted as $\boldsymbol{X} \oplus \boldsymbol{F}_{l-1}$ for simplicity. $\boldsymbol{X}_{l}^{\prime}$ is illustrated in Fig. 3. The transfer-based AK-LS-SVM is used in $L_{l}$, which leverages the source model in $L_{l-1}$ to have $\lambda_{l} \boldsymbol{w}_{l-1}$, which is represented as below:

$$
\begin{array}{ll}
\min J\left(\boldsymbol{w}_{l}, b_{l}\right)= & \frac{1}{2}\left\|\boldsymbol{w}_{l}-\lambda_{l} \boldsymbol{w}_{l-1}\right\|^{2}+\frac{C_{l}}{2} \sum_{i=1}^{N} \xi_{l i}^{2} \\
\text { s.t. } & y_{i}=\boldsymbol{w}_{l}^{T} \varphi\left(\boldsymbol{x}_{i}^{\prime}\right)+b_{l}+\xi_{l i} \\
& i=1,2, \ldots, N
\end{array}
$$

The corresponding Lagrangian $\mathcal{L}_{l}$ of Eq. (5) is

$$
\mathcal{L}_{l}\left(\boldsymbol{w}_{l}, b_{l}, \boldsymbol{\xi}_{l} ; \boldsymbol{\alpha}_{l}\right)=J\left(\boldsymbol{w}_{l}, b_{l}\right)+\sum_{i=1}^{N} \alpha_{l i}\left(y_{i}-\boldsymbol{w}_{l}^{T} \varphi\left(\boldsymbol{x}_{i}^{\prime}\right)-b_{l}-\xi_{l i}\right)
$$

where $\boldsymbol{\alpha}_{l} \in \mathbf{R}^{N}$ is the vector of all Lagrangian multipliers. With respect to $\boldsymbol{w}_{l}, \xi_{l i}, \alpha_{l i}$, the optimal condition can be calculated by

$$
\begin{gathered}
\frac{\partial \mathcal{L}_{l}}{\partial \boldsymbol{w}_{l}}=0 \Rightarrow \boldsymbol{w}_{l}=\lambda_{l} \boldsymbol{w}_{l-1}+\sum_{i=1}^{n} \alpha_{l i} \varphi\left(\boldsymbol{x}_{i}^{\prime}\right) \\
\frac{\partial \mathcal{L}_{l}}{\partial \xi_{l i}}=0 \quad \Rightarrow \xi_{l i}=\alpha_{l i} / C_{l} \\
\frac{\partial \mathcal{L}_{l}}{\partial \alpha_{l i}}=0 \Rightarrow y_{i}=\boldsymbol{w}_{l}^{T} \varphi\left(\boldsymbol{x}_{i}^{\prime}\right)+b_{l}+\xi_{l i}
\end{gathered}
$$

Note that the optimal solution $\boldsymbol{w}_{l}$ in Eq. (10) is given by the sum of the source model scaled by parameter $\lambda_{l}$ and a new model is built on the new data inputs. When $\lambda_{l}$ is 0 , $\boldsymbol{w}_{l}$ returns to the original formulation in a traditional AK-LSSVM model; no learned knowledge can be leveraged from the previous source model.

Combining Eq. (10), Eq. (11) with Eq. (12), we obtain:

$$
\sum_{i=1}^{N} \alpha_{l i} \varphi\left(\boldsymbol{x}_{i}^{\prime}\right)^{T} \varphi\left(\boldsymbol{x}_{j}^{\prime}\right)+b_{l}+\alpha_{l i} / C_{l}=y_{i}-\lambda_{l} \boldsymbol{w}_{l-1}^{T} \varphi\left(\boldsymbol{x}_{i}^{\prime}\right)
$$

The above equation can be written in matrix form: 
Layer $\mathbf{L}_{\mathrm{L}}$

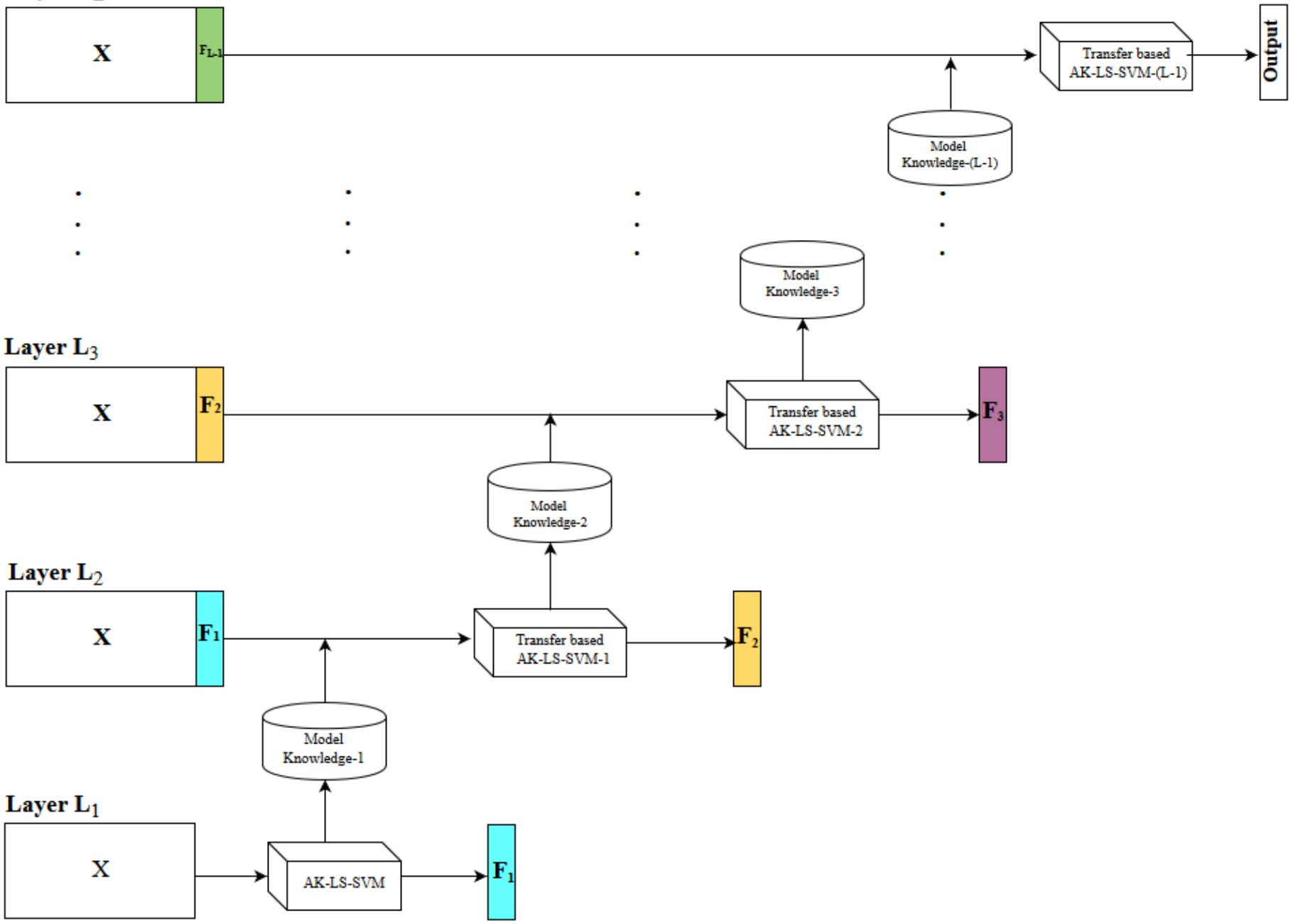

Fig. 2: Hierarchical architecture and learning process of DTA-LS-SVM

\begin{tabular}{|c|c|c|c|c|c|}
\hline Input & \multicolumn{5}{|c|}{ Features } \\
\cline { 2 - 6 } $\boldsymbol{X}_{l}^{\prime}$ & 1 & 2 & $\cdots$ & $d$ & $d+1$ \\
\hline $\boldsymbol{x}_{1}^{\prime}$ & & & & & $f_{l-1}\left(\boldsymbol{x}_{1}\right)$ \\
\hline $\boldsymbol{x}_{2}^{\prime}$ & & & & & $f_{l-1}\left(\boldsymbol{x}_{2}\right)$ \\
\hline$\vdots$ & & & & & $\vdots$ \\
\hline $\boldsymbol{x}_{N}^{\prime}$ & & & & & $f_{l-1}\left(\boldsymbol{x}_{N}\right)$ \\
\hline
\end{tabular}

Original data input $\boldsymbol{X} \quad$ Augmented feature space $\boldsymbol{F}_{l-1}$

Fig. 3: Augmented space $\boldsymbol{X}_{l}^{\prime}$ of $\boldsymbol{X}$

$$
\left[\begin{array}{cc}
\widetilde{\boldsymbol{K}}_{l}+C_{l}^{-1} \mathbf{I} & \mathbf{1} \\
\mathbf{1}^{T} & 0
\end{array}\right]\left[\begin{array}{c}
\boldsymbol{\alpha}_{l} \\
b_{l}
\end{array}\right]=\left[\begin{array}{c}
\boldsymbol{Y}-\lambda_{l} \tilde{\boldsymbol{Y}}_{l} \\
0
\end{array}\right]
$$

where $\widetilde{\boldsymbol{K}}_{l}=\left[\boldsymbol{K}\left(\boldsymbol{x}_{i}^{\prime}, \boldsymbol{x}_{j}^{\prime}\right)\right]_{N * N}, \boldsymbol{I}$ is a diagonal matrix with unity diagonal entries, $\boldsymbol{Y}$ is the actual labels of training samples, and $\widetilde{\boldsymbol{Y}}_{l}$ is the predicted labels of training samples that are obtained from the source model, i.e. $\boldsymbol{Y}=\left[y_{1} ; \cdots ; y_{N}\right]$,
$\tilde{\boldsymbol{Y}}_{l}=\left[y_{l 1}^{\prime} ; \cdots ; y_{l N}^{\prime}\right]=\left[\boldsymbol{w}_{l-1}^{T} \varphi\left(\boldsymbol{x}_{l 1}^{\prime}\right) ; \cdots ; \boldsymbol{w}_{l-1}^{T} \varphi\left(\boldsymbol{x}_{l N}^{\prime}\right)\right]=$ $\left[\sum_{i=1}^{N} \alpha_{(l-1) i} \boldsymbol{K}\left(\boldsymbol{x}_{(l-1) i}, \boldsymbol{x}_{l 1}^{\prime}\right), \cdots, \sum_{i=1}^{N} \alpha_{(l-1) i} \boldsymbol{K}\left(\boldsymbol{x}_{(l-1) i}, \boldsymbol{x}_{l N}^{\prime}\right)\right]$.

Here $\widetilde{\boldsymbol{Y}}_{l}$ can be obtained in a kernel form as long as the previous model $\boldsymbol{w}_{l-1}$ and the current model $\boldsymbol{w}_{l}$ use the same kernel. Therefore, for the safe use of the adopted Gaussian additive kernel, $\delta$ in each model must be the same.

$\mathbf{H}_{l}$ represents the first matrix on the left-hand side of Eq. (14); the model parameters can be calculated simply using a matrix inversion:

$$
\left[\begin{array}{c}
\boldsymbol{\alpha}_{l} \\
b_{l}
\end{array}\right]=\mathbf{Q}\left[\begin{array}{c}
\boldsymbol{Y}-\lambda_{l} \tilde{\boldsymbol{Y}}_{l} \\
0
\end{array}\right]
$$

where $\mathbf{Q}_{l}=\mathbf{H}_{l}^{-1}$. It can be observed that $\lambda_{l}, \boldsymbol{\alpha}_{l}$ and $b_{l}$ can be calculated accordingly from Eq. (15), and $\boldsymbol{w}_{l}$ and $b_{l}$ from Eq. (10) and Eq. (12), respectively.

In order to find the optimal value for parameter $\lambda_{l}$ efficiently and effectively, we propose a fast leave-one-out crossvalidation method for parameter tuning. 
$\mathbf{H}_{l}$ is decomposed into its block representation and the first row and the first column are isolated, i.e.,

$$
\mathbf{H}_{l}=\left[\begin{array}{cc}
\widetilde{\boldsymbol{K}_{l}}+C_{l}^{-1} \mathbf{I} & \mathbf{1} \\
\mathbf{1}^{T} & 0
\end{array}\right]=\left[\begin{array}{cc}
h_{11 l} & \mathbf{h}_{1 l}^{T} \\
\mathbf{h}_{1 l} & \mathbf{H}_{(-1) l}
\end{array}\right]
$$

$\boldsymbol{\alpha}_{l(-i)}$ and $b_{l(-i)}$ represent the model parameters in the $i$-th iteration of the leave-one-out cross validation procedure. In the first iteration where the first training sample is excluded, we have

$$
\left[\begin{array}{c}
\boldsymbol{\alpha}_{(-1) l} \\
b_{(-1) l}
\end{array}\right]=\mathbf{Q}_{(-1) l}\left[\begin{array}{c}
\boldsymbol{Y}_{(-1)}-\lambda_{l} \tilde{\boldsymbol{Y}}_{(-1) l} \\
0
\end{array}\right]
$$

where $\mathbf{Q}_{(-1) l}=\mathbf{H}_{(-1) l}^{-1}$ and $\boldsymbol{Y}_{(-1)}=\left(y_{2}, y_{3}, \ldots, y_{N}, 0\right)^{T}$. We denote the predicted label on the $i$-th sample excluded from the training dataset by $\tilde{y}_{i l}$, and the predicted label for the first training sample becomes

$$
\begin{aligned}
\tilde{y}_{1 l} & =\mathbf{h}_{1 l}^{T}\left[\begin{array}{c}
\boldsymbol{\alpha}_{(-1) l} \\
b_{(-1) l}
\end{array}\right]+\lambda_{l} \widetilde{\boldsymbol{Y}}_{l} \\
& =\mathbf{h}_{1 l}^{T} \mathbf{Q}_{(-1) l}\left(\boldsymbol{Y}_{(-1)}-\lambda_{l} \widetilde{\boldsymbol{Y}}_{(-1)}\right)+\lambda_{l} \widetilde{\boldsymbol{Y}}_{l}
\end{aligned}
$$

Considering the last $N$ equations in Eq. (14), we get $\left[\begin{array}{ll}\mathbf{h}_{1 l} & \mathbf{H}_{(-1) l}\end{array}\right]\left[\begin{array}{ll}\boldsymbol{\alpha}_{l}^{T} & b_{l}\end{array}\right]^{T}=\left(\boldsymbol{Y}_{(-1)}-\lambda_{l} \widetilde{\boldsymbol{Y}}_{(-1)}\right)$, and

$$
\begin{aligned}
\tilde{y}_{1 l}= & \mathbf{h}_{1 l}^{T} \mathbf{Q}_{(-1) l}\left[\mathbf{h}_{1 l} \mathbf{H}_{(-1) l}\right]\left[\alpha_{1 l}, \cdots, \alpha_{N l}, b_{l}\right]^{T}+\lambda_{l} \widetilde{\boldsymbol{Y}}_{(-1)} \\
& =\mathbf{h}_{1}^{T} \mathbf{Q}_{l(-1)} \mathbf{h}_{1} \alpha_{1}+\mathbf{h}_{1}^{T}\left[\alpha_{2 l}, \cdots, \alpha_{N l}, b_{l}\right]^{T}+\lambda_{l} \widetilde{\boldsymbol{Y}}_{(-1)}
\end{aligned}
$$

From Eq. (14), the first equation of the system is $y_{1}-$ $\lambda_{l} \boldsymbol{y}_{1(-1) l}=h_{11 l} \alpha_{1 l}+\mathbf{h l}_{1}^{T}\left[\alpha_{2 l}, \alpha_{3 l}, \cdots, \alpha_{N l}, b_{l}\right]^{T}$, and hence $\tilde{y}_{1}=y_{1}-\alpha_{1 l}\left(h_{11 l}-\mathbf{h}_{1 l}^{T} \mathbf{Q}_{(-1) l} \mathbf{h}_{1 l}\right)$. Finally, by using $\mathbf{Q}_{l}=$ $\mathbf{H}_{(-1) l}$ and the block matrix inversion lemma we can obtain

$$
\mathbf{Q}_{l}=\left[\begin{array}{cc}
v^{-1} & -v^{-1} \mathbf{h}_{1 l} \mathbf{Q}_{(-1) l} \\
\mathbf{Q}_{(-1) l}+v^{-1} \mathbf{Q}_{(-1) l} \mathbf{h}_{1 l}^{T} \mathbf{h}_{1 l} \mathbf{Q}_{(-1) l} & -v^{-1} \mathbf{Q}_{(-1) l} \mathbf{h}_{1 l}^{T}
\end{array}\right]
$$

where $v=h_{11 l}-\mathbf{h}_{1 l}^{T} \mathbf{Q}_{(-1) l} \mathbf{h}_{1 l}$. Since the system of linear equations in Eq. (14) is insensitive to permutations of the ordering of the equations, then

$$
\tilde{y}_{i l}=y_{i}-\alpha_{i l} / \mathbf{Q}_{i i l}
$$

By defining $\left[\boldsymbol{\alpha}_{l}^{\prime T}, b_{l}^{\prime}\right]^{T}=\mathbf{Q}_{l}\left[\boldsymbol{y}^{T}, 0\right],\left[\boldsymbol{\alpha}_{l}^{\prime \prime T}, b_{l}^{\prime \prime}\right]=$ $\mathbf{Q}_{l}\left[\boldsymbol{Y}^{T}, 0\right]$, and $\boldsymbol{\alpha}_{l}=\boldsymbol{\alpha}_{l}^{\prime}-\lambda_{l} \boldsymbol{\alpha}_{l}^{\prime \prime}$, then we can obtain

$$
\tilde{y}_{i l}=y_{i}-\frac{\alpha_{i l}^{\prime}}{\mathbf{Q}_{i i l}}+\frac{\lambda_{l} \alpha_{i l}^{\prime \prime}}{\mathbf{Q}_{i i l}}
$$

It can be seen from Eq. (22) that $\boldsymbol{\alpha}_{l}$ and $\lambda_{l}$ have a linear relationship, which means that after determining $\lambda_{l}$, the learning model can be obtained as well. The optimal $\lambda_{l}$ is supposed to keep the same sign of $\tilde{y}_{i l}$ and $y_{i}$ for all training samples. However, it might cause many local minima issues due to non-convex formulation. Thus, in the end the following loss function is adopted, which is similar to the hinge loss:

$$
l\left(\tilde{y}_{i l}, y_{i}\right)=\left|1-\tilde{y}_{i l} y_{i}\right|_{+}=\left|y_{i} \frac{\alpha_{i l}^{\prime}-\lambda_{l} \alpha_{i l}^{\prime \prime}}{\mathbf{Q}_{i i l}}\right|_{+}
$$

where $|x|_{+}=\max \{0, x\}$. This is a convex upper bound to the leave-one-out misclassification loss, and it prefers solutions in which $\tilde{y}_{i}$ has an absolute value equal to or larger than 1 and the same sign as $y_{i}$. Finally, the objective function is

$$
\begin{array}{ll} 
& \sum_{i=i}^{N} l\left(\tilde{y}_{i l}, y_{i}\right) \\
\text { s.t } & 0 \leq \lambda_{l} \leq D
\end{array}
$$

where $D$ is a constant. A regularization based on this can induce numerical stability. This optimization process can be implemented by a projected sub-gradient descent algorithm, and the pseudocode in given in Algorithm 1. After obtaining $\lambda_{l}, \boldsymbol{\alpha}_{l}$ and $b_{l}$ can be calculated accordingly from Eq. (15), and then $\boldsymbol{w}_{l}$ and $b_{l}$ from Eq. (10), Eq. (11) and Eq. (12).

As a result, we can obtain the decision function $f_{l}\left(\boldsymbol{x}_{i l}^{\prime}\right)=$ $\boldsymbol{w}_{l}^{T} \varphi\left(\boldsymbol{x}_{i l}^{\prime}\right)+b_{l}$ on $L_{l}(l \geq 2)$, and the predicted label vector $\boldsymbol{F}_{l}=\left(f_{l}\left(\boldsymbol{x}_{1 l}^{\prime}\right), f_{l}\left(\boldsymbol{x}_{2 l}^{\prime}\right), \cdots, f_{l}\left(\boldsymbol{x}_{N l}^{\prime}\right)\right)$ for $\boldsymbol{X}_{l}^{\prime}$. The processing layer continues to be added until the prediction accuracy performance has no improvement or the improvement is negligible, (i.e., $\left\|\boldsymbol{F}_{l}-\boldsymbol{F}_{l-1}\right\|_{F}^{2}<\epsilon$ ). Here, the complete learning algorithm of the proposed classifier DTA-LS-SVM is given in Algorithm 2 that outputs the final decision function. Please note that in this work we select the parameter $C_{l}$ from comparatively big intervals, i.e., $C_{l} \in\{1,10,50,100,150,200,250,500\}$, to guarantee diversities between the modules from adjacent layers. However $C_{l}$ can also be selected from different intervals depending upon the piratical situations.

In DTA-LS-SVM, transfer learning is embedded from the second layer. Referring to Eq. (10), we can observe that the classification on the $l$-th $(l>=2)$ layer is in fact achieved in a way like a combination of multiple kernel functions from different layers. According to the excellent generalization performances of multi-kernel classifiers [32]-[34], the module from the second layer in the proposed approach is supposed to obtain a better generalization performance than the previous one. In this sense, the learning process under such a stacked architecture tends to be greedy. Our experiments show that normally $L=3,4$ or 5 is an appropriate reference for small or medium datasets. If $L$ is too big, it might lead to overfitting issues.

The proposed classifier DTA-LS-SVM can be extended to explain the multi-classification tasks. The one-against-all strategy may be used to find the multiple decision functions that separate one class from the remaining classes. In the end, the predicted label of the new input data sample $\boldsymbol{x}_{i}$ is determined by $\max _{k=1, \cdots, M} y_{k}\left(\boldsymbol{x}_{i}\right)$, where $\mathrm{M}$ denotes the number of the classes.

\section{Computational complexity}

One highlight of the proposed classifier DTA-LS-SVM is its fast computational ability of the leave-one-out cross validation in the deep architecture. Its computational cost can be represented as $O\left(N^{3}+(L-1)\left(N^{3}+N\right)\right)$. First it includes the traditional AK-LS-SVM model construction on the first layer. Therefore, the complexity of this part is $O\left(N^{3}\right)$. From the layer $(l \geq 2)$, the computational cost of each module consists of two parts. The first part includes the calculation of the 

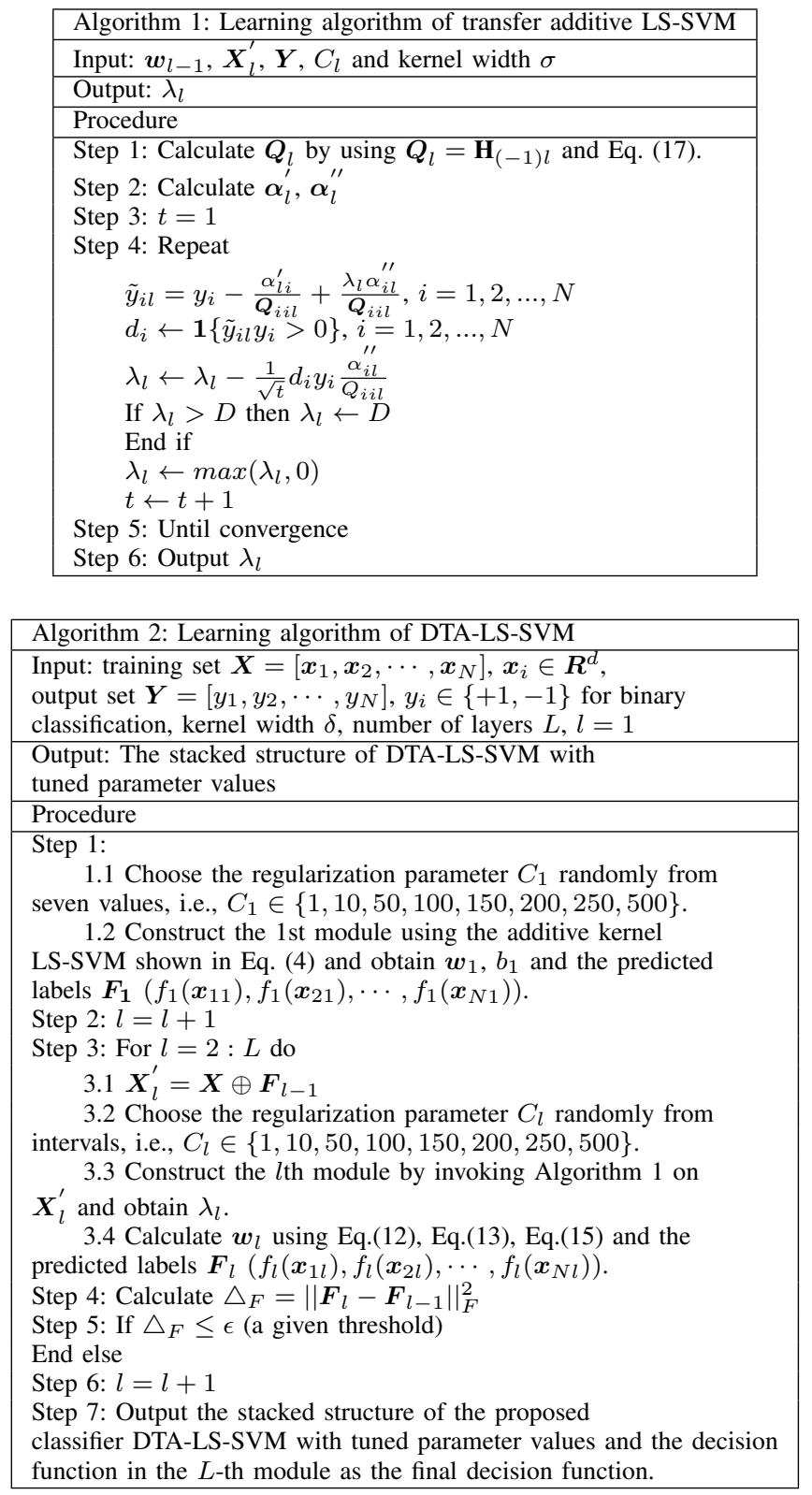

matrix $\mathbf{Q}_{l}$ by the inverse related to the training set on the $L_{l}$, in which the corresponding computational complexity is $O\left(N^{3}\right)$. The second part includes the computational complexity of each iteration in Algorithm 1 to optimize Eq. (24), which can be represented as $O(N)$. Therefore, the entire computational cost of DTA-LS-SVM becomes $O\left(N^{3}+(L-1)\left(N^{3}+N\right)\right)=$ $O\left(L N^{3}+(L-1) N\right)$.

Let us consider the traditional leave-one-out cross-validation strategy on SVM. Theoretically, it takes $O\left(N^{3}\right)$ to train a SVM. By using specific speed-up strategies [35], the training time can be accelerated to $O(N)-O\left(N^{2.3}\right)$ such that the leave-one-out cross validation time on SVM becomes $O(N * N)-O\left(N * N^{2.3}\right)=O\left(N^{2}\right)-O\left(N^{3.3}\right)$. Considering the grid search for generalization parameter $C$ ( $s_{1}$ grid values) and kernel width $\sigma$ ( $s_{2}$ grid values), the computational complexity of SVM becomes $s_{1} s_{2} O\left(N^{2}\right)-s_{1} s_{2} O\left(N^{3.3}\right)$. In general, $s_{1}$ and $s_{2}$ are normally greater than 3 in the experiments, and the number $L$ of layers in DTA-LS-SVM is small $(3 \leq L \leq 5)$.
Therefore, although it seems that the computational complexity of SVM may be less than that of DTA-LS-SVM, our experiments reveal that the actual running time of SVM with grid search actually is much longer than that of DTA-LS-SVM.

In terms of LS-SVM, the computational complexity to train a LS-SVM is $O\left(N^{3}\right)$ due to the calculation of the matrix $\mathbf{Q}$ by the inverse of $\mathbf{H}$. Therefore, the computational complexity of leave-one-out cross validation on LS-SVM becomes $s_{1} s_{2} O\left(N * N^{3}\right)=s_{1} s_{2} O\left(N^{4}\right)$. Moreover, LS-SVM could use the fast leave-one-out cross validation strategy discussed in Subsection III-B. Referring to Eq. (22) and Eq. (23) with $\lambda_{l}$ equal to 0 , the computational complexity of LS-SVM could be accelerated into $s_{1} s_{2} O\left(N^{3}+N\right)$. In summary, the proposed classifier DTA-LS-SVM has a superior advantage in the running speed, compared with SVM and LS-SVM.

\section{Extension on imbalanced datasets}

The proposed classifier DTA-LS-SVM is based on the AKLS-SVM framework. However, it is often the case in many real-world scenarios that the datasets are imbalanced. This is especially true when dealing with datasets involving medical problems. For example, a dataset will be imbalanced due to far more cases of a diagnosis of malignancy within cancer cases compared to those that are benign or cancer free. We found that DTA-LS-SVM can be naturally extended to its cost-sensitive or imbalanced version such that our method is also suitable for imbalanced datasets.

The problem with imbalanced datasets is that the decision boundary tends to get too close to the positive class (i.e., minority class). Therefore, the decision boundary needs to be pushed away from positive instances. One solution is to give different error costs to the positive and negative classes [36]. There are several ways to achieve this goal. However in this work we only change the objective functions of AK-LS-SVM into Eq. (25) for an imbalanced dataset:

$$
\begin{array}{ll}
\min _{\boldsymbol{w}, b} J(\boldsymbol{w}, b)= & \frac{1}{2}\|\boldsymbol{w}\|^{2}+\frac{C}{2}\left[\frac{N^{-}}{N} \sum_{i=1}^{N^{+}} \xi_{i}^{2}+\frac{N^{+}}{N} \sum_{i=N^{+}+1}^{N} \xi_{i}^{2}\right] \\
\text { s.t. } & y_{i}=\boldsymbol{w}^{T} \varphi\left(\boldsymbol{x}_{i}\right)+b+\xi_{i}
\end{array}
$$

where $N^{+}$and $N^{-}$represents the numbers of samples in the positive and negative classes respectively, in the $N$ samples. I.e., when Eq. (25) is applied to AK-LS-SVM, we term DTALS-SVM as iDTA-LS-SVM for an imbalanced dataset.

Similar to the mathematical derivations from Eq. (9) to Eq. (15), we easily find that only $\mathbf{H}_{l}$ in the first matrix in Eq. (14) needs to be changed into the following representation:

$$
\mathbf{H}_{l}=\left[\begin{array}{cc}
\widetilde{\boldsymbol{K}}_{l}+C_{l}^{-1} \mathbf{E} & \mathbf{1} \\
\mathbf{1}^{T} & 0
\end{array}\right]
$$


where

$$
\mathbf{E}=\left(\begin{array}{cccccc}
\frac{N^{-}}{N} & 0 & 0 & 0 & 0 & 0 \\
0 & \ddots & 0 & 0 & 0 & 0 \\
0 & 0 & \frac{N^{-}}{N} & 0 & 0 & 0 \\
0 & 0 & 0 & \frac{N^{+}}{N} & 0 & 0 \\
0 & \ddots & 0 & 0 & \ddots & 0 \\
0 & 0 & 0 & 0 & 0 & \frac{N^{+}}{N}
\end{array}\right)
$$

and the remaining derivations remain the same such that iDTA-LS-SVM can be applied on imbalanced datasets. By comparing $\mathbf{H}_{l}$ in Eq. (26) with the first matrix in Eq. (14), we can find that only the difference is between $\mathbf{E}$ in Eq. (26) and $\mathbf{I}$ in Eq. (14). Only under the condition that $N^{-}$equals $N^{+}, \mathbf{E}$ degenerates into I such that iDTA-LS-SVM can handle balanced datasets as well. In addition, it is obvious that iDTALS-SVM has the same computational complexity of leave-oneout cross validation as that for DTA-LS-SVM.

\section{EXPERIMENTS AND RESULTS}

In the experiments, the proposed classifiers DTA-LS-SVM and IDTA-LS-SVM were evaluated on balanced and imbalanced UCI datasets and one real world dataset. In order to make the comparison fair, their classification performances are compared with those using the traditional LS-SVM and SVM with additive kernels. During the data preparation process, all values in the datasets were normalized. For any original UCI datasets with missing data, the records with missing values were first removed and then the processed datasets were used in the experiments. To evaluate methods on balanced datasets, the accuracy and F-score were used as metrics. On imbalanced datasets, only the F-score was used as a metric. This considers for example, that with an imbalance ratio 99:1, a method labelling each data input with a positive class is 99\% accurate, but it is useless as a classifier to find out the negative class. Therefore, metrics precision and recall were adopted for imbalanced datasets. Precision is defined as the number of correct positive results divided by the number of all positive results, while recall is defined as the number of correct positive results divided by the number of positive results that should have been found. The F-score is a weighted average of the precision and recall.

$$
\begin{gathered}
\text { Precision }=\frac{\text { true positive }}{\text { true positive }+ \text { false positive }} \\
\text { Recall }=\frac{\text { true positive }}{\text { true positive }+ \text { false negative }} \\
\text { F-score }=2 * \frac{\text { precision } * \text { recall }}{\text { precision }+ \text { recall }}
\end{gathered}
$$

The F-score was used to measure the performances on imbalanced datasets in Lin et al. [37], Zou et al. [38] and Tan [39]. In this work, this metric was also used to evaluate iDTALS-SVM and comparative methods. In the experiments, each dataset was randomly split into the corresponding training and testing sets in the ratio 7:3. This process was repeated 10 times such that every sample from the dataset might have a chance
TABLE I: UCI DATASETS DESCRIPTION

\begin{tabular}{|c|c|c|c|c|}
\hline Type & Dataset & Sample size & Feature & Class(\%) \\
\hline \multirow{4}{*}{ Imbalanced } & breast cancer & 683 & 9 & $\begin{array}{l}65.52 \\
34.48\end{array}$ \\
\cline { 2 - 5 } & Pima Indians & 768 & 8 & $\begin{array}{l}65.02 \\
34.98\end{array}$ \\
\cline { 2 - 5 } & Indians Liver & 579 & 10 & $\begin{array}{l}71.50 \\
28.50\end{array}$ \\
\cline { 2 - 5 } Balanced & \multirow{2}{*}{ Australian } & 690 & 14 & $\begin{array}{l}44.50 \\
55.50\end{array}$ \\
\cline { 2 - 5 } & \multirow{2}{*}{ diabetic } & \multirow{2}{*}{1151} & 19 & $\begin{array}{l}53.08 \\
46.92\end{array}$ \\
\cline { 2 - 5 } & \multirow{2}{*}{ credit approval } & \multirow{2}{*}{653} & 15 & $\begin{array}{l}45.33 \\
54.67\end{array}$ \\
\cline { 2 - 5 } & \multirow{2}{*}{ mammographic } & \multirow{2}{*}{830} & 5 & $\begin{array}{l}48.55 \\
51.45\end{array}$ \\
\hline
\end{tabular}

to be used in the training and testing sets. In the end, the mean and standard deviation of metrics(s) on training and testing sets were calculated. All the experiments were implemented using 64-bit MATLAB on a computer with an Intel Core i5-6300 $2.40 \mathrm{GHz}$ CPU and 8.00GB RAM.

\section{A. UCI public datasets}

In this subsection, DTA-LS-SVM and iDTA-LS-SVM with the traditional LS-SVM and SVM using additive kernels are compared. Seven public UCI datasets were adopted in which three were imbalanced and four were balanced. These UCI datasets are summarized in Table I.

In the experiments, different additive kernels were tried to find the most suitable one for DTA-LS-SVM, iDTA-LSSVM and the comparative methods on each dataset. Here only the experimental results using the selected kernel (Gaussian additive kernel) are displayed. For DTA-LS-SVM and iDTA-LS-SVM, the number of modules is set to 3 or 4 due to the small or medium sample size of the adopted datasets. $\epsilon$ is set to $0.1 . \delta$ is set to be the average value of the standard deviations for all respective features. For the comparative methods, the grid search algorithm with 10fold cross-validation was used to find the optimal values for parameters $C$ and $\delta$ which could give the best performances. The values of $\{1,10,50,100,150,200,250,500\}$ and $\{0.1,1,5,10,20,50,100,150,200\}$ were searched for $C$ and $\delta$, respectively in the experiments. Here only the performances using the optimal parameters are displayed.

Table II shows the experimental results of DTA-LS-SVM and the comparative methods on imbalanced datasets. Table III shows the experimental results of iDTA-LS-SVM and the comparative methods on balanced datasets. During the training process of both DTA-LS-SVM and iDTA-LS-SVM classifiers, the first module (i.e., the first layer) was constructed to obtain the training accuracy after comparing the predicted and actual labels. If the training accuracy of the adjacent higher layer was improving continuously, one more layer was added and training continued. If the training accuracy of the adjacent layer remained the same or even dropped, the process was stopped. To elaborate, one complete training process on the Australian dataset is described. For this dataset, 
TABLE II: PERFORMANCE RESUlTS ON BALANCED UCI DATASETS

\begin{tabular}{|c|c|c|c|c|c|c|c|}
\hline \multirow{3}{*}{ Datasets } & \multirow{3}{*}{ Metrics } & \multicolumn{6}{|c|}{ Performances } \\
\hline & & \multicolumn{2}{|c|}{ DTA-LS-SVM } & \multicolumn{2}{|c|}{ LS-SVM } & \multicolumn{2}{|c|}{ SVM } \\
\hline & & training & testing & training & testing & training & testing \\
\hline \multirow{2}{*}{ Australian } & Accuracy & $0.8936 \pm 0.0099$ & $0.8678 \pm 0.0212$ & $0.8583 \pm 0.0098$ & $0.8500 \pm 0.0266$ & $0.8589 \pm 0.0075$ & $0.8572 \pm 0.0174$ \\
\hline & F1-score & $0.9050 \pm 0.0091$ & $0.8766 \pm 0.0215$ & $0.8665 \pm 0.0060$ & $0.8630 \pm 0.0193$ & $0.8620 \pm 0.0082$ & $0.8619 \pm 0.0190$ \\
\hline \multirow{2}{*}{ diabetic } & Accuracy & $0.7720 \pm 0.0096$ & $0.7395 \pm 0.0197$ & $0.7391 \pm 0.0221$ & $0.7220 \pm 0.0242$ & $0.7275 \pm 0.0068$ & $0.7188 \pm 0.0194$ \\
\hline & F1-score & $0.7668 \pm 0.0163$ & $0.7368 \pm 0.0202$ & $0.7434 \pm 0.0380$ & $0.7262 \pm 0.0281$ & $0.7262 \pm 0.0112$ & $0.7153 \pm 0.0194$ \\
\hline \multirow{2}{*}{$\begin{array}{c}\text { credit } \\
\text { approval }\end{array}$} & Accuracy & $0.8985 \pm 0.0075$ & $0.8786 \pm 0.0161$ & $0.8611 \pm 0.0138$ & $0.8536 \pm 0.0203$ & $0.8678 \pm 0.0087$ & $0.8648 \pm 0.0230$ \\
\hline & F1-score & $0.9065 \pm 0.0095$ & $0.8895 \pm 0.0154$ & $0.8683 \pm 0.0097$ & $0.8632 \pm 0.0205$ & $0.8684 \pm 0.0079$ & $0.8682 \pm 0.0192$ \\
\hline \multirow{2}{*}{ mammographic } & Accuracy & $0.8231 \pm 0.0078$ & $0.8273 \pm 0.0233$ & $0.8155 \pm 0.0103$ & $0.8121 \pm 0.0313$ & $0.8189 \pm 0.0116$ & $0.8104 \pm 0.0349$ \\
\hline & F1-score & $0.8227 \pm 0.0102$ & $0.8321 \pm 0.0266$ & $0.8172 \pm 0.0173$ & $0.8167 \pm 0.0357$ & $0.8181 \pm 0.0120$ & $0.8137 \pm 0.0350$ \\
\hline
\end{tabular}

TABLE III: PERFORMANCE RESULTS ON IMBALANCED UCI DATASETS

\begin{tabular}{|c|c|c|c|c|c|c|}
\hline \multirow{3}{*}{ Datasets } & \multicolumn{6}{|c|}{ Performances } \\
\hline & \multicolumn{2}{|c|}{ iDTA-LS-SVM } & \multicolumn{2}{|c|}{ LS-SVM } & \multicolumn{2}{|c|}{ SVM } \\
\hline & training & testing & training & testing & training & testing \\
\hline breast cancer & $0.9861 \pm 0.0064$ & $0.9801 \pm 0.0026$ & $0.9760 \pm 0.0074$ & $0.9738 \pm 0.0069$ & $0.9806 \pm 0.0041$ & $0.9728 \pm 0.0123$ \\
\hline Pima Indians & $0.8748 \pm 0.0167$ & $\mathbf{0 . 8 3 5 9} \pm \mathbf{0 . 0 0 9 7}$ & $0.8401 \pm 0.0131$ & $0.8284 \pm 0.0317$ & $0.8336 \pm 0.0084$ & $0.8285 \pm 0.0248$ \\
\hline Indians liver & $0.8438 \pm 0.0829$ & $0.8403 \pm 0.0083$ & $0.8390 \pm 0.0166$ & $0.7986 \pm 0.0580$ & $0.8343 \pm 0.0080$ & $0.8323 \pm 0.0188$ \\
\hline
\end{tabular}

TABle IV: Performance Results on the Australian Dataset with Different Ratios of Training and Testing DATA

\begin{tabular}{|c|c|c|c|c|c|c|c|c|c|}
\hline \multirow{3}{*}{ Australia } & \multirow{3}{*}{ Metrics } & \multicolumn{8}{|c|}{ Performances } \\
\hline & & \multicolumn{2}{|c|}{$6: 4$} & \multicolumn{2}{|c|}{$5: 5$} & \multicolumn{2}{|c|}{$4: 6$} & \multicolumn{2}{|c|}{$3: 7$} \\
\hline & & training & testing & training & testing & training & testing & training & testing \\
\hline \multirow{2}{*}{$\begin{array}{c}\text { DTA-LS- } \\
\text { SVM }\end{array}$} & Accuracy & $0.9086 \pm 0.0087$ & $0.8572 \pm 0.0111$ & $0.9009 \pm 0.0148$ & $0.8577 \pm 0.0110$ & $0.9047 \pm 0.0215$ & $0.8589 \pm 0.0141$ & $0.9043 \pm 0.0175$ & $0.8551 \pm 0.0149$ \\
\hline & F1-score & $0.9081 \pm 0.0083$ & $0.8716 \pm 0.0125$ & $0.9123 \pm 0.0121$ & $0.8727 \pm 0.0098$ & $0.9124 \pm 0.0199$ & $0.8742 \pm 0.0131$ & $0.9132 \pm 0.0171$ & $0.8703 \pm 0.0132$ \\
\hline \multirow{2}{*}{ LS-SVM } & Accuracy & $0.8425 \pm 0.0384$ & $0.8417 \pm 0.0431$ & $0.8417 \pm 0.0598$ & $0.8304 \pm 0.0692$ & $0.8413 \pm 0.0544$ & $0.8283 \pm 0.0559$ & $0.8449 \pm 0.0695$ & $0.8265 \pm 0.0762$ \\
\hline & F1-score & $0.8586 \pm 0.0200$ & $0.8595 \pm 0.0281$ & $0.8631 \pm 0.0307$ & $0.8546 \pm 0.0396$ & $0.8600 \pm 0.0272$ & $0.8519 \pm 0.0325$ & $0.8651 \pm 0.0337$ & $0.8496 \pm 0.0418$ \\
\hline \multirow{2}{*}{ SVM } & Accuracy & $0.8621 \pm 0.0148$ & $0.8504 \pm 0.0221$ & $0.8649 \pm 0.0143$ & $0.8487 \pm 0.0139$ & $0.8717 \pm 0.0202$ & $0.8498 \pm 0.0176$ & $0.8797 \pm 0.0251$ & $0.8478 \pm 0.0161$ \\
\hline & F1-score & $0.8663 \pm 0.0139$ & $0.8564 \pm 0.0194$ & $0.8716 \pm 0.0150$ & $0.8529 \pm 0.0126$ & $0.8788 \pm 0.0176$ & $0.8563 \pm 0.0164$ & $0.8871 \pm 0.0242$ & $0.8556 \pm 0.0155$ \\
\hline
\end{tabular}

TABle V: Performance Results on the Diabetic Dataset with Different Ratios of Training and Testing DATA

\begin{tabular}{|c|c|c|c|c|c|c|c|c|c|}
\hline \multirow{3}{*}{ Diabetic } & \multirow{3}{*}{ Metrics } & \multicolumn{8}{|c|}{ Performances } \\
\hline & & \multicolumn{2}{|c|}{$6: 4$} & \multicolumn{2}{|c|}{$5: 5$} & \multicolumn{2}{|c|}{$4: 6$} & \multicolumn{2}{|c|}{$3: 7$} \\
\hline & & training & testing & training & testing & training & testing & training & testing \\
\hline \multirow{2}{*}{$\begin{array}{c}\text { DTA-LS- } \\
\text { SVM }\end{array}$} & Accuracy & $0.7990 \pm 0.0082$ & $0.7386 \pm 0.0139$ & $0.8028 \pm 0.0125$ & $0.7241 \pm 0.0164$ & $0.8052 \pm 0.0121$ & $0.7159 \pm 0.0132$ & $0.8183 \pm 0.0150$ & $0.7076 \pm 0.0129$ \\
\hline & F1-score & $0.7974 \pm 0.0090$ & $0.7331 \pm 0.0117$ & $0.7986 \pm 0.0154$ & $0.7127 \pm 0.0197$ & $0.8084 \pm 0.0186$ & $0.7071 \pm 0.0132$ & $0.8115 \pm 0.0240$ & $0.6965 \pm 0.0162$ \\
\hline \multirow{2}{*}{ LS-SVM } & Accuracy & $0.7445 \pm 0.0216$ & $0.7180 \pm 0.0208$ & $0.7431 \pm 0.0166$ & $0.7163 \pm 0.0245$ & $0.7315 \pm 0.0286$ & $0.6991 \pm 0.0237$ & $0.7209 \pm 0.0143$ & $0.6815 \pm 0.0237$ \\
\hline & F1-score & $0.7451 \pm 0.0333$ & $0.7270 \pm 0.0250$ & $0.7377 \pm 0.0409$ & $0.7096 \pm 0.0338$ & $0.7355 \pm 0.0402$ & $0.7021 \pm 0.0333$ & $0.7268 \pm 0.0482$ & $0.6770 \pm 0.0412$ \\
\hline \multirow{2}{*}{ SVM } & Accuracy & $0.7330 \pm 0.0134$ & $0.7015 \pm 0.0199$ & $0.7297 \pm 0.0136$ & $0.7038 \pm 0.0145$ & $0.7193 \pm 0.0100$ & $0.6987 \pm 0.0151$ & $0.7075 \pm 0.0216$ & $0.6875 \pm 0.0210$ \\
\hline & F1-score & $0.7350 \pm 0.0219$ & $0.7038 \pm 0.0147$ & $0.7262 \pm 0.0226$ & $0.6936 \pm 0.0276$ & $0.7339 \pm 0.0154$ & $0.6965 \pm 0.0095$ & $0.7004 \pm 0.0355$ & $0.6733 \pm 0.0432$ \\
\hline
\end{tabular}

TABLE VI: PERformance Results on the credit approval DATASET With Different RATIOS of Training And TESTING DATA

\begin{tabular}{|c|c|c|c|c|c|c|c|c|c|}
\hline \multirow{3}{*}{$\begin{array}{c}\text { credit } \\
\text { approval }\end{array}$} & \multirow{3}{*}{ Metrics } & \multicolumn{8}{|c|}{ Performances } \\
\hline & & \multicolumn{2}{|c|}{$6: 4$} & \multicolumn{2}{|c|}{$5: 5$} & \multicolumn{2}{|c|}{$4: 6$} & \multicolumn{2}{|c|}{$3: 7$} \\
\hline & & training & testing & training & testing & training & testing & training & testing \\
\hline \multirow{2}{*}{$\begin{array}{c}\text { DTA-LS- } \\
\text { SVM }\end{array}$} & Accuracy & $0.8985 \pm 0.0071$ & $0.8664 \pm 0.0115$ & $0.9110 \pm 0.0100$ & $0.8621 \pm 0.0116$ & $0.9031 \pm 0.0119$ & $0.8615 \pm 0.0112$ & $0.9190 \pm 0.0149$ & $0.8614 \pm 0.0126$ \\
\hline & F1-score & $0.9074 \pm 0.0075$ & $\mathbf{0 . 8 7 7 3} \pm \mathbf{0 . 0 1 2 2}$ & $0.9206 \pm 0.0107$ & $\mathbf{0 . 8 7 0 8} \pm \mathbf{0 . 0 1 2 4}$ & $0.9120 \pm 0.0107$ & $0.8720 \pm 0.0097$ & $0.9270 \pm 0.0134$ & $0.8687 \pm 0.0168$ \\
\hline \multirow{2}{*}{ LS-SVM } & Accuracy & $0.8509 \pm 0.0305$ & $0.8481 \pm 0.0556$ & $0.8426 \pm 0.0763$ & $0.8202 \pm 0.0890$ & $0.8295 \pm 0.0798$ & $0.8120 \pm 0.0997$ & $0.8508 \pm 0.0649$ & $0.8328 \pm 0.0630$ \\
\hline & F1-score & $0.8623 \pm 0.0182$ & $0.8608 \pm 0.0406$ & $0.8614 \pm 0.0382$ & $0.8411 \pm 0.0506$ & $0.8609 \pm 0.0396$ & $0.8405 \pm 0.0556$ & $0.8673 \pm 0.0363$ & $0.8560 \pm 0.0423$ \\
\hline \multirow[b]{2}{*}{ SVM } & Accuracy & $0.8681 \pm 0.0050$ & $0.8622 \pm 0.0127$ & $0.8696 \pm 0.0164$ & $0.8612 \pm 0.0169$ & $0.8778 \pm 0.0149$ & $0.8564 \pm 0.0108$ & $0.8677 \pm 0.0164$ & $0.8537 \pm 0.0193$ \\
\hline & F1-score & $0.8695 \pm 0.0059$ & $0.8628 \pm 0.0149$ & $0.8716 \pm 0.0159$ & $0.8625 \pm 0.0167$ & $0.8802 \pm 0.0167$ & $0.8604 \pm 0.0117$ & $0.8754 \pm 0.0172$ & $0.8600 \pm 0.0132$ \\
\hline
\end{tabular}

TABLE VII: PeRformance Results on the mammographic DATASET With Different RATIOS OF TRAining AND TESTING DATA

\begin{tabular}{|c|c|c|c|c|c|c|c|c|c|}
\hline \multirow{3}{*}{ mammographic } & \multirow{3}{*}{ Metrics } & \multicolumn{8}{|c|}{ Performances } \\
\hline & & \multicolumn{2}{|c|}{$6: 4$} & \multicolumn{2}{|c|}{$5: 5$} & \multicolumn{2}{|c|}{ 4:6 } & \multicolumn{2}{|c|}{$3: 7$} \\
\hline & & training & testing & training & testing & training & testing & training & testing \\
\hline DTA-LS- & Accuracy & $0.8325 \pm 0.0045$ & $0.8187 \pm 0.0171$ & $0.8234 \pm 0.0120$ & $0.8224 \pm 0.0118$ & $0.8241 \pm 0.0173$ & $0.8106 \pm 0.0138$ & $0.8261 \pm 0.0240$ & $0.8095 \pm 0.0165$ \\
\hline SVM & F1-score & $0.8317 \pm 0.0078$ & $0.8093 \pm 0.0149$ & $0.8214 \pm 0.0130$ & $0.8234 \pm 0.0132$ & $0.8216 \pm 0.0264$ & $0.8091 \pm 0.0213$ & $0.8275 \pm 0.0267$ & $0.8050 \pm \mathbf{0 . 0 1 7 1}$ \\
\hline \multirow{2}{*}{ LS-SVM } & Accuracy & $0.8213 \pm 0.0137$ & $0.8051 \pm 0.0173$ & $0.8212 \pm 0.0086$ & $0.8039 \pm 0.0181$ & $0.8250 \pm .0180$ & $0.8036 \pm 0.0186$ & $0.8309 \pm 0.0172$ & $0.8024 \pm 0.0149$ \\
\hline & F1-score & $0.8202 \pm 0.0166$ & $0.8017 \pm 0.0201$ & $0.8151 \pm 0.0081$ & $0.7988 \pm 0.0183$ & $0.8240 \pm 0.0202$ & $0.8006 \pm 0.0178$ & $0.8326 \pm 0.0209$ & $0.7975 \pm 0.0153$ \\
\hline \multirow{2}{*}{ SVM } & Accuracy & $0.8139 \pm 0.0125$ & $0.8060 \pm 0.0284$ & $0.8089 \pm 0.0124$ & $0.8000 \pm 0.0153$ & $0.8108 \pm 0.0197$ & $0.7972 \pm 0.0215$ & $0.8092 \pm 0.0277$ & $0.7914 \pm 0.0153$ \\
\hline & F1-score & $0.8165 \pm 0.0119$ & $0.8079 \pm 0.0264$ & $0.8135 \pm 0.0138$ & $0.7978 \pm 0.0159$ & $0.8110 \pm 0.0187$ & $0.8008 \pm 0.0189$ & $0.8052 \pm 0.0296$ & $0.7867 \pm 0.0234$ \\
\hline
\end{tabular}


TABLE VIII: Performance Results on the breast cancer DAtASET With DifFEREnT Ratios of Training AND TESTING DATA

\begin{tabular}{|c|c|c|c|c|c|c|c|c|}
\hline \multirow{3}{*}{ breast cancer } & \multicolumn{8}{|c|}{ F-score } \\
\hline & \multicolumn{2}{|c|}{$6: 4$} & \multicolumn{2}{|c|}{$5: 5$} & \multicolumn{2}{|c|}{$4: 6$} & \multicolumn{2}{|c|}{$3: 7$} \\
\hline & training & testing & training & testing & training & testing & training & testing \\
\hline iDTA-LS-SVM & $0.9818 \pm 0.0038$ & $0.9798 \pm 0.0053$ & $0.9821 \pm 0.0040$ & $0.9787 \pm 0.0044$ & $0.9859 \pm 0.0065$ & $0.9796 \pm 0.0031$ & $0.9874 \pm 0.0043$ & $0.9775 \pm 0.0053$ \\
\hline LS-SVM & $0.9735 \pm 0.0051$ & $0.9742 \pm 0.0115$ & $0.9684 \pm 0.0183$ & $0.9649 \pm 0.0236$ & $0.9750 \pm 0.0044$ & $0.9675 \pm 0.0111$ & $0.9745 \pm 0.0093$ & $0.9711 \pm 0.0057$ \\
\hline SVM & $0.9811 \pm 0.0042$ & $0.9711 \pm 0.0063$ & $0.9797 \pm 0.0081$ & $0.9749 \pm 0.0064$ & $0.9821 \pm 0.0042$ & $0.9726 \pm 0.0083$ & $0.9854 \pm 0.0088$ & $0.9718 \pm 0.0043$ \\
\hline
\end{tabular}

TABLE IX: Performance Results on the Pima Indians Dataset with Different Ratios of Training and Testing DATA

\begin{tabular}{|c|c|c|c|c|c|c|c|c|}
\hline \multirow{3}{*}{$\begin{array}{l}\text { Pima } \\
\text { Indians }\end{array}$} & \multicolumn{8}{|c|}{ F-score } \\
\hline & \multicolumn{2}{|c|}{$6: 4$} & \multicolumn{2}{|c|}{$5: 5$} & \multicolumn{2}{|c|}{$4: 6$} & \multicolumn{2}{|c|}{$3: 7$} \\
\hline & training & testing & training & testing & training & testing & training & testing \\
\hline iDTA-LS-SVM & $0.8665 \pm 0.0157$ & $0.8345 \pm 0.0171$ & $0.8697 \pm 0.0088$ & $0.8345 \pm 0.0066$ & $0.8700 \pm 0.0129$ & $0.8336 \pm 0.0121$ & $0.8918 \pm 0.0188$ & $0.8254 \pm 0.0088$ \\
\hline LS-SVM & $0.8399 \pm 0.0141$ & $0.8265 \pm 0.0239$ & $0.8409 \pm 0.0179$ & $0.8295 \pm 0.0205$ & $0.8360 \pm 0.0252$ & $0.8263 \pm 0.0189$ & $0.8385 \pm 0.0280$ & $0.8222 \pm 0.0178$ \\
\hline SVM & $0.8379 \pm 0.0104$ & $0.8185 \pm 0.0138$ & $0.8417 \pm 0.0175$ & $0.8198 \pm 0.0161$ & $0.8426 \pm 0.0150$ & $0.8174 \pm 0.0075$ & $0.8478 \pm 0.0134$ & $0.8141 \pm 0.0135$ \\
\hline
\end{tabular}

TABLE X: Performance Results on the Indians liver Dataset with Different Ratios of Training and Testing DATA

\begin{tabular}{|c|c|c|c|c|c|c|c|c|}
\hline \multirow{3}{*}{ Indians liver } & \multicolumn{8}{|c|}{ F-score } \\
\hline & \multicolumn{2}{|c|}{$6: 4$} & \multicolumn{2}{|c|}{$5: 5$} & \multicolumn{2}{|c|}{$4: 6$} & \multicolumn{2}{|c|}{$3: 7$} \\
\hline & training & testing & training & testing & training & testing & training & testing \\
\hline iDTA-LS-SVM & $0.8387 \pm 0.0104$ & $\mathbf{0 . 8 3 7 5} \pm \mathbf{0 . 0 1 3 5}$ & $0.8422 \pm 0.0085$ & $\mathbf{0 . 8 3 7 3} \pm \mathbf{0 . 0 0 7 8}$ & $0.8382 \pm 0.0113$ & $0.8336 \pm 0.0096$ & $0.8353 \pm 0.0177$ & $\mathbf{0 . 8 2 9 4} \perp \mathbf{0 . 0 1 7 7}$ \\
\hline LS-SVM & $0.9197 \pm 0.0583$ & $0.8285 \pm 0.0153$ & $0.8714 \pm 0.0537$ & $0.8117 \pm 0.0212$ & $0.8850 \pm 0.0779$ & $0.7995 \pm 0.0542$ & $0.9203 \pm 0.0760$ & $0.7813 \pm 0.0542$ \\
\hline SVM & $0.8369 \pm 0.0106$ & $0.8294 \pm 0.0160$ & $0.8420 \pm 0.0133$ & $0.8253 \pm 0.0133$ & $0.8410 \pm 0.0175$ & $0.8288 \pm 0.0117$ & $0.8363 \pm 0.0203$ & $0.8226 \pm 0.0088$ \\
\hline
\end{tabular}

the training (testing) accuracy is 0.8589 (0.8173), respectively, after the first module, and 0.8734 (0.8365), respectively, after the second module (i.e., the second layer). Since the accuracies are increasing, training of the third and forth module continued, and $0.8880(0.8702)$ accuracy for the third module and $0.8734(0.8365)$ accuracy for the fourth module was obtained. After the third module, a decrease in the accuracy can be seen. Therefore the accuracy performances on the third module are used, which indeed outperforms the comparative methods. From the performance results shown in Table II, DTA-LS-SVM obtains considerably higher testing accuracies than those using the comparative methods on the adopted balanced UCI datasets. In terms of the experimental results on the imbalanced datasets displayed in Table III, iDTA-LS-SVM maintains the advantage on the F-scores compared to other methods. From these two tables, the performance increases from DTA-LS-SVM and iDTA-LS-SVM are not dramatic. However compared to LS-SVM, it becomes consistently better. In some cases, LS-SVM performs worse than SVM, but DTALS-SVM and iDTA-LS-SVM always perform better. Overall, the proposed classifiers DT-AK-LS-SVM and iDTA-LS-SVM exhibited good generalization performances on both balanced and imbalanced datasets.

Moreover, to observe the behavior of the proposed classifiers, we divided the training and testing sets at different ratios on each UCI dataset to evaluate their classification performances. The training and testing sets ratios were set to $6: 4,5: 5,4: 6$ and $3: 7$ on each UCI datasets. Table IVVII display the experimental results of DTA-LS-SVM and the comparative methods on all the balanced datasets in terms of accuracy and F score. Tables VIII-X contain the experimental results of iDTA-LS-SVM and the comparative methods for all the imbalanced datasets, in terms of F-scores. On each table, the best results have been highlighted in bold.
In terms of training accuracy and F-score, when changing the ratios, DTA-LS-SVM and iDTA-LS-SVM do not exhibit an increasing trend as seen in LS-SVM and SVM when the size of the training set increases. The reason might be that DTALS-SVM and iDTA-LS-SVM essentially are multiple kernel combination methods, which have a stronger representation capability for smaller training datasets. Importantly, it is easily seen that after changing the ratios of training and testing sets, the proposed classifiers DTA-LS-SVM and iDTA-LS-SVM still outperform LS-SVM and SVM on the testing accuracies of all seven datasets. This can be seen, for example, in Table V for the mammographic dataset at the 5:5 ratio; DTA-LS-SVM not only performs the best in terms of overall accuracy but also has the highest $\mathrm{F}$ score on the testing sets. Another example is for the imbalanced Pima Indians dataset in Table IX in which iDTA-LS-SVM maintains an advantage over the comparative methods in terms of the F-score on the testing set. Overall, the experimental results demonstrate that DTA-LS-SVM and iDTA-LS-SVM are tolerant to the change of training sample size and is a favorable choice for balanced and imbalanced datasets in terms of the generalization capability.

In terms of the average training and testing time, Table. XI in the 7:3 ratio case shows that DTA-LS-SVM and iDTALS-SVM take much less training time compared to the other methods. As explained in subsection III-C, the running time of DTA-LS-SVM and iDTA-LS-SVM is the summation of the running time in each module plus the running time for the parameter tuning of $\lambda_{l}(l=1,2, \cdots, L)$ using a fast leaveone-out cross validation strategy. The regularization parameter $C_{l}(l=1,2, \cdots, L)$ in each module and the kernel width $\delta$ can be randomly chosen instead of using model selection strategy, which also significantly reduces the running time. Conversely, LS-SVM and SVM take much more time to produce the optimal values for $C$ and $\delta$ by searching from 8 and 9 grid 
values respectively which is much more computationally expensive. After this comparison, DTA-LS-SVM and iDTA-LSSVM exhibited superior advantages in running times compared to other methods.

\section{B. Real-world dataset: the community healthcare dataset}

In this subsection, a real community healthcare dataset was used in the experiments to investigate performances of the proposed classifiers DTA-LS-SVM and iDTA-LS-SVM.

This dataset was collected in the nurse-led PolyU-Henry G. Leong Mobile Integrative Health Care Centre (MIHC) [40] in August 2013. It contains 444 patient records with 33 features, such as demographic, socio-economic, social relationship, and social participation data. Additionally, information on the patients' health history, such as smoking and drinking habits, chronic illnesses, and data from a series of health assessments with descriptions was included. Due to the nature of both the tests performed on the mobile clinic and the patients' themselves, some values in the dataset were missing. For example, language barriers affect the communications between nurses and patients. The missing values in the dataset were filled in by using the $K$-nearest neighbor (KNN) imputation method [41] with their corresponding values from the nearest-neighbour columns using the corresponding Euclidean distance.

The label information is the overall quality of life (QOL) score on a 1-5 scale of 444 patients obtained using the World Heath Organization questionnaire on quality of life: from the Hong Kong short version (WHOQOL-BREF(HK)) framework [42] [43]. After analysis, the proportion of the two constructed classes ("poor" and "good") was 122 and 322, respectively. We aim to construct a classifier to predict the QOL of elderly patients ("poor" or "good") using these 33 features from this MIHC dataset.

Since the two classes were originally imbalanced, iDTALS-SVM was used on the datasets, and its classification performance was compared with those using DTA-LS-SVM, LS-SVM, and SVM. In addition, for iDTA-LS-SVM and DTALS-SVM, experiments were divided into two parts for more detailed comparisons:

Part (a): In layer 1 of DTA-LS-SVM and iDTA-LS-SVM, the complete portion of the MIHC dataset, which had no missing data was used to train an AK-LS-SVM model. From layer 2 , the whole dataset after imputation and its corresponding predictions from the previous model were used as the new data input.

Part (b): In layer 1 of DTA-LS-SVM and iDTA-LS-SVM, the whole $M I H C$ dataset was used after imputation as the input.

The number of processing layers in both DTA-LS-SVM and iDTA-LS-SVM was set to 3 in these experiments. The ratio of training and testing data sets was set to 7:3. The classification accuracies and the running time of DTA-LSSVM, iDTA-LS-SVM and the comparative methods on the training and testing datasets are listed in Table XII, XIII, and XIV. From the experimental results, iDTA-LS-SVM in Part (b) has achieved the best testing accuracy (0.7425) and a F-score of 0.8553 compared to DTA-LS-SVM, LS-SVM and SVM on the MIHC dataset. In Part (a), it achieved an accuracy of 0.7331 and an F-score of 0.8492 , which are still higher than other methods. It shows that iDTA-LS-SVM is more suitable for classification on imbalanced datasets. The traditional LS-SVM had the worst accuracy and F-score on the testing dataset, iDTA-LS-SVM based on the combination of several AK-LS-SVM modules achieved the best accuracy. We believe that this performance improvement has arisen from the stacked generalization principle and the transfer learning mechanism. In terms of the running time, DTA-LS-SVM and iDTA-LS-SVM remains superior over LS-SVM and SVM.

In addition, we also notice that in the experimental results of DTA-LS-SVM and iDTA-LS-SVM, both accuracy and Fscore on the testing data set are higher than those on the training data set. There might be two reasons to explain these results. First, DTA-LS-SVM and iDTA-LS-SVM used the stacked hierarchical architecture to enhance generalization performances and this is reflected in the obtained results. Second, in the data preparation, the KNN imputation method was used to fill in missing values in the MIHC dataset. Following this procedure, the distributions in the training and testing sets might be mismatched. Referring to Gonzalez and Abu-Mostafa's [44] conclusions, we postulate that mismatched distributions also occurred in this experiment, which may have caused a higher testing accuracy and F-score in DTA-LS-SVM and iDTA-LS-SVM.

\section{Statistical analysis}

In order to detect significant differences among the experimental results of the proposed methods and the comparative methods, we also carried out the Friedman ranking test followed by Holm post-hoc test [45], [46] for multiple comparisons on the testing sets of four balanced and four imbalanced datasets in which the ratio of training and testing data sets is $7: 3$. The Friedman ranking test was used to evaluate whether there was a statistically significant difference among these methods. The null hypothesis is that there is no statistically difference. If the $p$-value is smaller than 0.5 , the null hypothesis is rejected. The Holm post-hoc test was used to further verify if there was a statistical difference between the best Friedman ranking method and each remaining method. We used $\alpha=0.05$ as the level of confidence in all cases. First, we conducted two Friedman ranking tests to assess whether there are significant differences between (1) DTA-LS-SVM and the comparative methods on four balanced UCI datasets in terms of accuracy and F1-score; (2) iDTA-LS-SVM and the comparative methods on three imbalanced UCI datasets and one real-world TRUS dataset in terms of F1-score. Here, experimental results of both Part (a) and (b) on the TRUS dataset were included.

The ranking results of Friedman test (1) in terms of accuracy and F1-score are shown in Tables. XV and XVII, respectively. The results reveal that there are significant differences in the both performance metrics between DTA-LS-SVM and other comparative methods. Then we conducted the Holm post-hoc tests to compare the best ranking method DTA-LS-SVM with LS-SVM and SVM in terms of accuracy and F1-score, and 
TABLE XI: Training and Testing Time (SEConds) on Seven UCi Datasets

\begin{tabular}{|c|c|c|c|c|c|c|c|}
\hline \multirow{2}{*}{ Type } & \multirow{2}{*}{ Datasets } & \multicolumn{6}{|c|}{ Running time } \\
\cline { 3 - 8 } & & (i)DTA-LS-SVM & \multicolumn{2}{c|}{ LS-SVM } & \multicolumn{2}{c|}{ SVM } \\
\cline { 3 - 8 } & training & testing & training & testing & training & testing \\
\hline \multirow{3}{*}{ Imbalanced } & breast cancer & 2.278 & 1.979 & 8776.1 & 1.399 & 19553 & 2.498 \\
\cline { 2 - 8 } & Pima Indians & 2.731 & 2.376 & 10512 & 1.447 & 28950 & 3.018 \\
\cline { 2 - 8 } & Indians liver & 1.886 & 1.679 & 6488.1 & 1.249 & 13304 & 2.087 \\
\cline { 2 - 8 } Balanced & australian & 3.472 & 2.935 & 12103 & 1.835 & 22059 & 3.209 \\
\cline { 2 - 8 } & diabetic & 12.698 & 10.600 & 73863 & 6.568 & 129790 & 12.943 \\
\cline { 2 - 8 } & credit approval & 3.638 & 2.782 & 11660 & 2.203 & 18138 & 3.190 \\
\cline { 2 - 8 } & mammographic & 2.279 & 1.933 & 8340.3 & 1.034 & 29387 & 3.212 \\
\hline
\end{tabular}

TABLE XII: Performance Results on the MIHC Dataset using iDTA-LS-SVM

\begin{tabular}{|c|c|c|c|c|}
\hline \multirow{4}{*}{ MIHC } & \multicolumn{4}{|c|}{ Performances } \\
\hline & \multicolumn{4}{|c|}{ iDTA-LS-SVM } \\
\hline & \multicolumn{2}{|c|}{ Part (a) } & \multicolumn{2}{|c|}{ Part (b) } \\
\hline & training & testing & training & testing \\
\hline Accuracy & $0.7261 \pm 0.0094$ & $0.7331 \pm 0.0218$ & $0.7210 \pm 0.0102$ & $\mathbf{0 . 7 4 2 5} \pm \mathbf{0 . 0 2 6 6}$ \\
\hline F1-score & $0.8413 \pm 0.0063$ & $0.8492 \pm 0.0147$ & $0.8358 \pm 0.0100$ & $\mathbf{0 . 8 5 5 3} \pm \mathbf{0 . 0 2 3 3}$ \\
\hline
\end{tabular}

TABle XIII: Performance Results on the MiHC Dataset using DTA-LS-SVM and the Other Comparative METHODS

\begin{tabular}{|c|c|c|c|c|c|c|c|c|}
\hline \multirow{4}{*}{ MIHC } & \multicolumn{8}{|c|}{ Performances } \\
\hline & \multicolumn{4}{|c|}{ DTA-LS-SVM } & \multirow{2}{*}{\multicolumn{2}{|c|}{ LS-SVM }} & \multirow{2}{*}{\multicolumn{2}{|c|}{ SVM }} \\
\hline & & & & & & & & \\
\hline & training & testing & training & testing & training & testing & training & testing \\
\hline Accuracy & $0.7203 \pm 0.0133$ & $0.7266 \pm 0.0309$ & $0.7294 \pm 0.0146$ & $0.7224 \pm 0.0391$ & $0.7510 \pm 0.0452$ & $0.7050 \pm 0.0398$ & $0.7274 \pm 0.0166$ & $0.7201 \pm 0.0384$ \\
\hline F1-score & $0.8374 \pm 0.0090$ & $0.8380 \pm 0.0205$ & $0.8430 \pm 0.0101$ & $0.8379 \pm 0.0262$ & $0.8452 \pm 0.0103$ & $0.8268 \pm 0.0439$ & $0.8435 \pm 0.0057$ & $0.8341 \pm 0.0133$ \\
\hline
\end{tabular}

TABLE XIV: Training And Testing Time (Seconds) on the MIHC Dataset

\begin{tabular}{|c|c|c|c|c|c|c|c|c|c|c|c|c|}
\hline \multirow{3}{*}{ MIHC } & \multicolumn{4}{|c|}{ iDTA-LS-SVM } & \multicolumn{4}{|c|}{ DTA-LS-SVM } & \multirow{2}{*}{\multicolumn{2}{|c|}{ LS-SVM }} & \multirow{2}{*}{\multicolumn{2}{|c|}{ SVM }} \\
\hline & \multicolumn{2}{|c|}{ Part (a) } & \multicolumn{2}{|c|}{ Part (b) } & \multicolumn{2}{|c|}{ Part (a) } & \multicolumn{2}{|c|}{ Part (b) } & & & & \\
\hline & training & testing & traning & testing & training & testing & training & testing & training & testing & training & testing \\
\hline Time (s) & 5.530 & 5.201 & 5.837 & 5.437 & 4.343 & 4.019 & 4.673 & 4.217 & 4950.6 & 2.398 & 1157.8 & 4.183 \\
\hline
\end{tabular}

TABLE XV: AVERAGE RANKINGS OF DTA-LS-SVM AND THE Comparative Methods on Balanced Datasets in TERMS OF ACCURACY $(p$-VALUE $=0.049787)$

\begin{tabular}{|c|c|}
\hline Methods & Ranking \\
\hline DTA-LS-SVM & 1 \\
\hline LS-SVM & 2.5 \\
\hline SVM & 2.5 \\
\hline
\end{tabular}

TABLE XVI: HOLM POST-HOC COMPARISON RESULTS FOR DTA-LS-SVM AND THE OTHER METHODS IN TERMS OF ACCURACY WITH $\alpha=0.05$

\begin{tabular}{|c|c|c|c|c|}
\hline$i$ & Methods & $z$-value & $p$-value & Holm $=\alpha / i$ \\
\hline 2 & LS-SVM & 2.12132 & 0.033895 & 0.025 \\
\hline 1 & SVM & 2.12132 & 0.033895 & 0.05 \\
\hline
\end{tabular}

presented the results in Tables. XVI and XVIII where the methods are ranked according to the obtained $z$-values. Holm post-hoc test rejects the hypothesis of equivalence for the methods with $p<\alpha / i$. According to the results in these tables, we know that DTA-LS-SVM is at least comparable to LSSVM and SVM on balanced datasets in terms of accuracy; and is at least comparable to LS-SVM and statistically outperforms SVM on balanced datasets in terms of F1-score.

The ranking results of Friedman test (2) are shown in Table. XIX. The results reveal that there are significant differences
TABLE XVII: AVERAGE RANKINGS OF DTA-LS-SVM AND THE Comparative Methods on BalanCEd Datasets IN TERMS OF F1-SCORE $(p$-VALUE $=0.038774)$

\begin{tabular}{|c|c|}
\hline Methods & Ranking \\
\hline DTA-LS-SVM & 1 \\
\hline LS-SVM & 2.25 \\
\hline SVM & 2.75 \\
\hline
\end{tabular}

TABLE XVIII: Holm Post-Hoc COMPARISON Results FOR DTA-LS-SVM AND THE OTHER METHODS IN TERMS OF F1-SCORE WITH $\alpha=0.05$

\begin{tabular}{|c|c|c|c|c|}
\hline$i$ & Methods & $z$-value & $p$-value & Holm $=\alpha / i$ \\
\hline 2 & SVM & 2.474874 & 0.013328 & 0.025 \\
\hline 1 & LS-SVM & 1.767767 & 0.0771 & 0.05 \\
\hline
\end{tabular}

in terms of F1-score between iDTA-LS-SVM and the other comparative methods. Then we conducted the Holm post-hoc test to compare the best ranking method iDTA-LS-SVM with LS-SVM and SVM, and presented the results in Table. XX. According to the obtained results, iDTA-LS-SVM outperforms the other methods on imbalanced datasets in terms of F1-score in our experiments.

In summary, the proposed methods are at least comparable to or even better than LS-SVM and SVM in terms of accuracy and/or F1-score with the faster learning speed. 
TABLE XIX: AVERAGE RANKINGS OF iDTA-LS-SVM AND THE COMPARATIVE Methods ON IMBALANCED DatasetS IN TERMS OF F1-SCORE $(p$-VALUE $=0.022371)$

\begin{tabular}{|c|c|}
\hline Methods & Ranking \\
\hline iDTA-LS-SVM & 1 \\
\hline LS-SVM & 2.6 \\
\hline SVM & 2.4 \\
\hline
\end{tabular}

TABLE XX: Holm Post-Hoc COMPARISON RESUltS FOR iDTA-LS-SVM AND THE OTHER Methods With $\alpha=0.05$

\begin{tabular}{|c|c|c|c|c|}
\hline$i$ & Methods & $z$-value & $p$-value & Holm $=\alpha / i$ \\
\hline 2 & LS-SVM & 2.529822 & 0.011412 & 0.025 \\
\hline 1 & SVM & 2.213594 & 0.026857 & 0.05 \\
\hline
\end{tabular}

\section{CONCLUSIONS AND FURTHER STUDY}

AK-LS-SVM has been applied recently in many classification tasks. In this work, to improve its generalization performance and learning speed, we proposed the novel classifier DTA-LS-SVM to be applied to balanced datasets, which follows the stacked generalization principle and transfer learning mechanism, and its extended version iDTA-LSSVM on imbalanced datasets. DTA-LS-SVM and iDTA-LSSVM stack multiple AK-LS-SVMs layer-by-layer where the prediction from the previous module becomes one additional feature space together with the original data inputs to train the next module of the next layer. Moreover, transfer learning is embedded into the deep architecture to guarantee consistency across adjacent modules, and thus the classification capability of the module at the higher layer can be further enhanced. In addition, transfer learning based on AK-LS-SVM can perfectly formulate a fast leave-one-out cross validation strategy for the learning parameter tuning such that even though the kernel width and the regularization parameter are randomly selected, the performance of the proposed method can remain outstanding. We compared the proposed method with the traditional LS-SVM and SVM using additive Gaussian kernels on seven public UCI datasets and one real world community healthcare dataset. The experimental results indicate that the proposed classifiers DTA-LS-SVM and iDTA-LS-SVM have the superior advantages on the generalization performance and the running time. Moreover, the experimental results also reveal that both DTA-LS-SVM and iDTA-LS-SVM have the potential to be applied in a real world application.

Even though the proposed methods show a promising performance, this study has some limitations that will be addressed in the future work. For example, DT-AK-LS-SVM has to use the same kernel in every processing layer. The proposed model will be investigated using different kernels in the stacked architecture to further improve the generalization performance.

\section{ACKNOWLEDGEMENT}

The work was supported by the Australian Research Council (ARC) under Discovery Grant DP140101366, and was also supported in part by the Hong Kong Research Grants Council (PolyU152040/16E), the Hong Kong Polytechnic University
(G-UC93, G-YBKX), the Tai Hung Fai Charitable Foundation and the YC Yu Scholarship for Centre for Smart Health.

\section{REFERENCES}

[1] G. C. Cawley, "Leave-one-out cross-validation based model selection criteria for weighted LS-SVMs," in Proc. 6th Joint Int. Conf. Neural Netw., Vancouver, BC, Canada, 2006, pp. 1661-1668.

[2] $\mathrm{H}$. Yang and J. Wu, "Practical large scale classification with additive kernels," in Proc. 4th Asian Conf. Mach. Learning, Singapore, 2012, pp. $523-538$.

[3] A. Vedaldi and A. Zisserman, "Efficient additive kernels via explicit feature maps," IEEE Trans. Pattern Anal. Mach. Intell, vol. 34, no. 3, pp. $480-492,2012$

[4] S. Maji, A. C. Berg, and J. Malik, "Efficient classification for additive kernel SVMs," IEEE Trans. Pattern Anal. Mach. Intell, vol. 35, no. 1, pp. $66-77,2013$.

[5] D. K. Duvenaud, H. Nickisch, and C. E. Rasmussen, "Additive gaussian processes," in Advances in Neural Inform. Process. Syst., 2011, pp. 226234.

[6] G. Wang, Z. Deng, and K.-S. Choi, "Tackling missing data in community health studies using additive LS-SVM classifier," IEEE J. Biomed. Health Inform., vol. PP, no. 99, 2016.

[7] C. M. Salgado, J. L. Viegas, C. S. Azevedo, M. C. Ferreira, S. M. Vieira, and J. M. da Costa Sousa, "Takagi-sugeno fuzzy modeling using mixed fuzzy clustering," IEEE Trans. Fuzzy Syst., 2016.

[8] B. Fan, X. Lu, and H.-X. Li, "Probabilistic inference-based least squares support vector machine for modeling under noisy environment," IEEE Trans. Syst., Man, Cybern., Syst, vol. 46, no. 12, pp. 1703-1710, 2016.

[9] L. Bi, O. Tsimhoni, and Y. Liu, "Using the support vector regression approach to model human performance," IEEE Trans. Syst., Man, Cybern., Syst and Humans, vol. 41, no. 3, pp. 410-417, 2011.

[10] L. Wang, Z. Liu, C. P. Chen, Y. Zhang, S. Lee, and X. Chen, "A UKFbased predictable SVR learning controller for biped walking," IEEE Trans. Syst., Man, Cybern., Syst, vol. 43, no. 6, pp. 1440-1450, 2013.

[11] A. Abdullah, R. C. Veltkamp, and M. A. Wiering, "An ensemble of deep support vector machines for image categorization," in Proc. 1st Int. Conf. Soft Comput. and Pattern Recognit., Malacca, Malaysia, 2009, pp. 301-306.

[12] Y. Tang, "Deep learning using linear support vector machines," arXiv preprint arXiv:1306.0239, 2013.

[13] A. Krizhevsky, I. Sutskever, and G. E. Hinton, "Imagenet classification with deep convolutional neural networks," in Advances in Neural Inform. Process. Syst., 2012, pp. 1097-1105.

[14] G. E. Hinton, "Deep belief networks," Scholarpedia, vol. 4, no. 5, p. 5947, 2009.

[15] R. Salakhutdinov and G. E. Hinton, "Deep boltzmann machines," in Proc. 12th Int. Conf. Artificial Intell. and Statistics, vol. 1, Clearwater, Florida, USA, 2009, p. 3

[16] M. Wang, H.-X. Li, X. Chen, and Y. Chen, "Deep learning-based model reduction for distributed parameter systems," IEEE Trans. Syst., Man, Cybern., Syst., vol. 46, no. 12, pp. 1664-1674, 2016.

[17] L. Deng and D. Yu, "Deep convex net: A scalable architecture for speech pattern classification," in Proc. 12th Ann. Int. Conf. Speech Comm. Assoc., Florence, Italy, 2011, pp. 2285-2288.

[18] D. H. Wolpert, "Stacked generalization," Neural Netw., vol. 5, no. 2, pp 241-259, 1992.

[19] L. Deng, G. Tur, X. He, and D. Hakkani-Tur, "Use of kernel deep convex networks and end-to-end learning for spoken language understanding," in Proc. 4th IEEE Workshop Spoken Language Tech., Miami, FL, USA, 2012, pp. 210-215.

[20] B. Hutchinson, L. Deng, and D. Yu, "Tensor deep stacking networks," IEEE Trans. Pattern Anal. Mach. Intel., vol. 35, no. 8, pp. 1944-1957, 2013.

[21] O. Vinyals, Y. Jia, L. Deng, and T. Darrell, "Learning with recursive perceptual representations," in Advances in Neural Inform. Process. Syst., 2012, pp. 2825-2833.

[22] S. J. Pan and Q. Yang, "A survey on transfer learning," IEEE Trans. Knowl. Data Eng., vol. 22, no. 10, pp. 1345-1359, 2010.

[23] W. Dai, Q. Yang, G.-R. Xue, and Y. Yu, "Boosting for transfer learning," in Proc. 24th Int. Mach. Learning Conf., Corvallis, OR, USA, 2007, pp. $193-200$.

[24] J. Jiang and C. Zhai, "Instance weighting for domain adaptation in NLP," in Proc. 45th Ann. Meeting Assoc. Computational Linguistics, vol. 7 , Prague, Czech Republic, 2007, pp. 264-271. 
[25] A. Quattoni, M. Collins, and T. Darrell, "Transfer learning for image classification with sparse prototype representations," in Proc. IEEE Int. Conf. Comput. Vis. and Pattern Recognit., Anchorage, AK, USA, 2008, pp. 1-8.

[26] C. Wang and S. Mahadevan, "Manifold alignment using procrustes analysis," in Proc. 25th Int. Conf. Mach. Learning, Helsinki, Finland, 2008, pp. 1120-1127.

[27] M. Stark, M. Goesele, and B. Schiele, "A shape-based object class model for knowledge transfer," in Proc. IEEE Int. Conf. Comput. Vis. and Pattern Recognit., 2009, pp. 373-380.

[28] J. Gao, W. Fan, J. Jiang, and J. Han, "Knowledge transfer via multiple model local structure mapping," in Proc. 14th ACM Int. Conf. Knowl. Discovery and Data Mining, Las Vegas, NV, USA, 2008, pp. 283-291.

[29] J. Lu, V. Behbood, P. Hao, H. Zuo, S. Xue, and G. Zhang, "Transfer learning using computational intelligence: a survey," Knowledge-Based Syst., vol. 80, pp. 14-23, 2015.

[30] D. Rafailidis and A. Nanopoulos, "Modeling users preference dynamics and side information in recommender systems," IEEE Trans. Syst., Man, Cybern., Syst, vol. 46, no. 6, pp. 782-792, 2016.

[31] Y. Bengio, G. Mesnil, Y. Dauphin, and S. Rifai, "Better mixing via deep representations," in Proc. 30th Int. Conf. Mach. Learning, 2013, pp. 552-560.

[32] G. R. Lanckriet, T. De Bie, N. Cristianini, M. I. Jordan, and W. S. Noble, "A statistical framework for genomic data fusion," Bioinformatics, vol. 20, no. 16, pp. 2626-2635, 2004.

[33] T. Senechal, V. Rapp, H. Salam, R. Seguier, K. Bailly, and L. Prevost, "Combining AAM coefficients with LGBP histograms in the multikernel SVM framework to detect facial action units," in Proc. 9th IEEE Int. Conf. Automatic Face \& Gesture Recognition and Workshops, Santa Barbara, California, USA, 2011, pp. 860-865.

[34] C.-Y. Yeh, C.-W. Huang, and S.-J. Lee, "A multiple-kernel support vector regression approach for stock market price forecasting," Expert Syst. with Applicat., vol. 38, no. 3, pp. 2177-2186, 2011.

[35] I.-H. Tsang, J.-Y. Kwok, and J. M. Zurada, "Generalized core vector machines," IEEE Trans. on Neural Netw., vol. 17, no. 5, pp. 1126-1140, 2006.

[36] R. OBatuwita and V. Palade, "Class imbalance learning methods for support vector machines," in Imbalanced Learning: Foundations, Algorithms, and Application, H. He and Y. Ma, Eds. John Wiley \& Sons, 2013, ch. 5, pp. 83-99.

[37] S.-J. Lin, C. Chang, and M.-F. Hsu, "Multiple extreme learning machines for a two-class imbalance corporate life cycle prediction," Knowledge-Based Syst., vol. 39, pp. 214-223, 2013.

[38] Q. Zou, S. Xie, Z. Lin, M. Wu, and Y. Ju, "Finding the best classification threshold in imbalanced classification," Big Data Research, vol. 5, pp. 2-8, 2016.

[39] S. Tan, "Neighbor-weighted K-nearest neighbor for unbalanced text corpus," Expert Syst. with Applicat., vol. 28, no. 4, pp. 667-671, 2005.

[40] K.-S. Choi, R. K. Wai, and E. Y. Kwok, "Healthcare information system: a facilitator of primary care for underprivileged elderly via mobile clinic," in Proc. 1st Int. Conf. Smart Health, Beijing, China, 2013, pp. $107-112$.

[41] G. E. Batista and M. C. Monard, "A study of k-nearest neighbour as an imputation method," in Proc. 2nd Int. Conf. Hybrid Intell. Syst., vol. 87, no. 48, Santiago, Chile, 2002.

[42] K. Leung, W. Wong, M. Tay, M. Chu, and S. Ng, "Development and validation of the interview version of the hong kong chinese whoqolbref," Quality of Life Research, vol. 14, no. 5, pp. 1413-1419, 2005.

[43] K. Leung, M. Tay, S. Cheng, and F. Lin, "Hong Kong Chinese version World Health Organization quality of life measure-abbreviated version," WHOQOL-BREF (HK), 1997.

[44] C. R. González and Y. S. Abu-Mostafa, "Mismatched training and test distributions can outperform matched ones," Neural Computation, vol. 27, no. 2, pp. 365-387, 2015.

[45] J. Demšar, "Statistical comparisons of classifiers over multiple data sets," J. of Mach. Learning Research, vol. 7, no. Jan, pp. 1-30, 2006.

[46] S. Garcia and F. Herrera, "An extension on 'statistical comparisons of classifiers over multiple data sets' for all pairwise comparisons," J. of Mach. Learning Research, vol. 9, no. Dec, pp. 2677-2694, 2008.

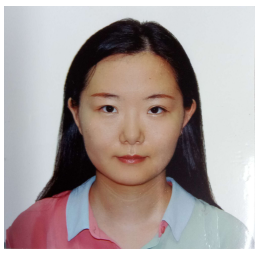

Guanjin Wang received both bachelor and Master degrees in Information Technology and Systems from Monash University, Australia in 2012 and 2014 respectively.

She is currently a joint Ph.D. student with the Faculty of Engineering and Information Technology, University of Technology Sydney, Broadway, NSW, Australia, and the School of Nursing, Hong Kong Polytechnic University, Hong Kong. Her current research interest includes machine learning, computational intelligence, and health informatics.

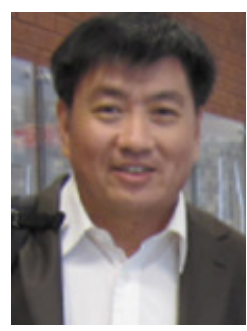

Guangquan Zhang received the Ph.D. degree in applied mathematics from the Curtin University of Technology, Perth, WA, Australia, in 2001.

$\mathrm{He}$ is currently an Associate Professor and the Director of the Decision Systems and e- Service Intelligence Laboratory, Centre for Artificial Intelligence, Faculty of Engineering and Information Technology, University of Technology Sydney, Broadway, NSW, Australia. He has authored four monographs, five reference books, and over 400 papers, including over 200 refereed journals. His current research interests include multiobjective and group decision making, decision support systems, fuzzy measure and optimization, concept drift, and uncertain information processing.

Dr. Zhang was a recipient of seven Australian Research Council Discovery Grants and several other research grants.

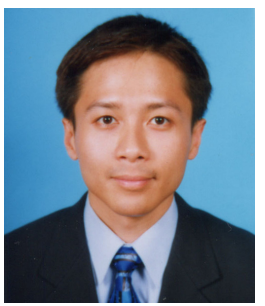

Kup-Sze Choi (M'97) received the Ph.D. degree in computer science and engineering from the Chinese University of Hong Kong, Hong Kong.

$\mathrm{He}$ is currently an Associate Professor with the School of Nursing, as well as the Director of the Centre for Smart Health and the PolyU-Henry G. Leong Mobile Integrative Health Centre, Hong Kong Polytechnic University, Hong Kong. His current research interests include virtual reality and artificial intelligence, and their applications in medicine and healthcare.

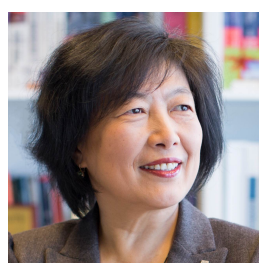

Jie Lu (SM'13) received the Ph.D. degree in information systems from the Curtin University of Technology, Perth, WA, Australia, in 2000.

She is currently a Distinguished Professor and the Associate Dean in research excellence with the Faculty of Engineering and Information Technology, and the Director of the Centre for Artificial Intelligence, University of Technology Sydney, Broadway, NSW, Australia. She has authored ten research books and over 400 papers in international journals and conferences. Her current research interests include decision support systems, fuzzy transfer learning, recommender systems, concept drift, and e-Service intelligence.

Dr. Lu was a recipient of eight Australian Research Council Discovery Grants. She serves as the Editor-in-Chief for Knowledge-Based Systems (Elsevier) and the International Journal of Computational Intelligence Systems (Atlantis). She is a fellow of IFSA. 